\title{
Standard model with a complex scalar singlet: Cosmological implications and theoretical considerations
}

\author{
Cheng-Wei Chiang, ${ }^{1,2,3,4, *}$ Michael J. Ramsey-Musolf, ${ }^{5,6, \dagger}$ and Eibun Senaha ${ }^{1,7, \ddagger}$ \\ ${ }^{1}$ Department of Physics, National Taiwan University, Taipei, Taiwan 10617, Republic of China \\ ${ }^{2}$ Institute of Physics, Academia Sinica, Taipei, Taiwan 11529, Republic of China \\ ${ }^{3}$ Physics Division, National Center for Theoretical Sciences, Hsinchu, Taiwan 30013, Republic of China \\ ${ }^{4}$ Kavli IPMU, University of Tokyo, Kashiwa 277-8583, Japan \\ ${ }^{5}$ Amherst Center for Fundamental Interactions, Department of Physics, \\ University of Massachusetts-Amherst Amherst, Massachusetts 01003, USA \\ ${ }^{6}$ California Institute of Technology, Pasadena, California 91125, USA \\ ${ }^{7}$ Center for Theoretical Physics of the Universe, Institute for Basic Science (IBS), Daejeon 34051, Korea
}

(Received 26 August 2017; published 8 January 2018)

\begin{abstract}
We analyze the theoretical and phenomenological considerations for the electroweak phase transition and dark matter in an extension of the standard model with a complex scalar singlet (cxSM). In contrast with earlier studies, we use a renormalization group improved scalar potential and treat its thermal history in a gauge-invariant manner. We find that the parameter space consistent with a strong first-order electroweak phase transition (SFOEWPT) and present dark matter phenomenological constraints is significantly restricted compared to results of a conventional, gauge-noninvariant analysis. In the simplest variant of the cxSM, recent LUX data and a SFOEWPT require a dark matter mass close to half the mass of the standard model-like Higgs boson. We also comment on various caveats regarding the perturbative treatment of the phase transition dynamics.
\end{abstract}

DOI: 10.1103/PhysRevD.97.015005

\section{INTRODUCTION}

Explaining the excess of matter over antimatter in the present Universe is a challenge and the interface of particle and nuclear physics with cosmology. It is well known that the standard model (SM) cannot account for the observed baryon asymmetry of the Universe (BAU) [1]

$$
Y_{B}=\frac{n_{B}}{s}=(8.59 \pm 0.11) \times 10^{-11} \quad(\text { Planck }),
$$

where $n_{B}(s)$ is the baryon number (entropy) density. While the electroweak (EW) sphalerons of the SM fulfil the first of the "Sakharov criteria" [2], the presence of baryon number (B) violating processes, the SM fails with regard to the remaining two requirements: sufficiently effective $C P$ violation and out-of-equilibrium dynamics, assuming $C P T$ symmetry is conserved.

\footnotetext{
*chengwei@phys.ntu.edu.tw

†jmm@physics.umass.edu

*senaha@ibs.re.kr
}

Published by the American Physical Society under the terms of the Creative Commons Attribution 4.0 International license. Further distribution of this work must maintain attribution to the author(s) and the published article's title, journal citation, and DOI. Funded by SCOAP.
A plethora of baryogenesis scenarios beyond the standard model (BSM) have been proposed to remedy these SM shortcomings. Among the most theoretically attractive and phenomenologically testable is electroweak baryogenesis (EWBG) [3] (for reviews, see Refs. [4-12]). EWBG proceeds via bubble nucleation during a first-order electroweak phase transition (EWPT), providing the needed outof-equilibrium conditions. BSM $C P$-violating interactions at the bubble walls generate a net density of left-handed fermions, biasing the EW sphalerons into the creation of a nonzero baryon number density that diffuses into the expanding bubble interiors. For a sufficiently strong firstorder electroweak phase transition (SFOEWPT), the sphaleron transitions in the bubble interiors are suppressed so as to preserve the generated baryon asymmetry.

The SM cannot accommodate a first-order EWPT since the observed Higgs boson is too heavy. Lattice computations indicate that the maximum mass for a first-order transition is 70-80 GeV [13-16]. An extended scalar sector from the SM, however, can allow a SFOEWPT for a mass of $125 \mathrm{GeV}$. The simplest such extensions involve the addition of gauge-singlet scalars. Such scalars may arise in a variety of contexts, such as the next-to-minimal supersymmetric standard model or $\mathrm{U}(1)^{\prime}$ extensions of the SM. By focusing on the role played by the associated singlet scalars, one may infer general features of the EWPT that 
are common to these and other scenarios without making reference to other model-specific details.

The possibilities for a SFOEWPT with an additional real singlet scalar (dubbed the "xSM" [17]), along with its phenomenological consequences for collider studies, have been studied extensively [18-29]. The xSM implies the existence of two neutral mass eigenstates, $H_{1,2}$, that are mixtures of the neutral doublet and real singlet. Signatures associated with a SFOEWPT include reduced SM-like Higgs signal strengths, modifications of the Higgs trilinear self-coupling, exotic decays of the SM-like Higgs boson, and resonant di-Higgs production. The last provides a particularly interesting opportunity not only for the LHC $[23,28]$ but also for a possible future higher-energy $p p$ collider $[26,29]$. Under some conditions, when the xSM assumes a $\mathbb{Z}_{2}$ symmetry, it may also provide a dark matter (DM) candidate with an associated vector boson fusion signature for the next-generation $p p$ collider [30].

In this work, we focus on the extension with one complex scalar singlet, the cxSM. This scenario was introduced in Ref. [31], where it was shown that one could obtain both the conditions for a SFOEWPT and a viable DM candidate even in the absence of a $\mathbb{Z}_{2}$ symmetry. The presence of a global U(1) symmetry that is both spontaneously and softly broken implies the existence of two mixed states $H_{1,2}$ of the xSM plus a pseudoscalar dark matter candidate $A$. A subsequent extensive study of the implications for vacuum stability and DM phenomenology was given in Ref. [32]. A less minimal version of the cxSM was also studied recently by the authors of Ref. [33] who also included a nonrenormalizable, $C P$-violating top quarkscalar interactions as needed to generate the BAU. For suitable choices of the model parameters, it appears possible to obtain both the observed BAU and DM relic density while satisfying present phenomenological constraints. ${ }^{1}$

Given the richness of this simple scenario, a more thorough study of open theoretical issues as well as phenomenological implications is in order. These issues include the following:

(i) To what extent do the conclusions of earlier studies hold when an appropriately gauge-invariant (GI) treatment of EWPT properties is performed?

(ii) To what extent can the renormalization scale dependence of computed EWPT properties be controlled?

(iii) What can one conclude about the possible thermal history of electroweak symmetry breaking (EWSB) in this scenario when a gauge-invariant and scaleinvariant treatment is performed?

(iv) To what extent does the gauge-invariant scale $\bar{v}(T)$ [35] associated with EWSB characterize the sphaleron energy $E_{\mathrm{sph}}(T)$ ? Is the latter simply proportional to the former?

\footnotetext{
${ }^{1}$ Reference [34] also considers a complex singlet extension that includes an additional neutral fermion as the dark matter candidate.
}

In addressing these issues, we perform a GI study of the cxSM EWPT dynamics following the framework of Ref. [35] and show how use of a renormalization group (RG)-improved version of the model significantly reduces the dependence on the renormalization scale. We also delineate various possibilities of the cxSM thermal history and analyze the effectiveness of baryon number preservation as a function of model parameters. Lastly, we connect these features to phenomenological signatures. We find that the null results for spin-independent DM-nucleus scattering obtained by the LUX collaboration [36] severely constrain the SFOEWPT-viable parameter space. We can say with confidence that only the finely tuned region for $m_{A} \approx m_{h} / 2$ remains viable, where $m_{A}$ and $m_{h}$ are the pseudoscalar dark matter and Higgs boson masses, respectively. While the parameter space for much larger values of $m_{A}$ (approaching $1 \mathrm{TeV}$ ) may also accommodate the LUX bounds while providing for a SFOEWPT, the remaining theoretical ambiguities associated with perturbative treatments of the EWPT dynamics render this possibility less certain. Our conclusions in this respect are less optimistic than those given in Ref. [33] that carried out a gauge-dependent treatment of the scalar effective potential. Although the latter study included a wider set of terms in the scalar potential that we consider below, a comparison of our GI, RG-improved analysis with the conventional treatment suggests that the SFOEWPT-viable parameter space associated with the wider set of operators used in Ref. [33] may be more restricted than indicated in that study.

The discussion of our analysis is organized as follows. In Sec. II, we introduce the scalar potential of the cxSM and analyze EWSB at $T=0$. In Sec. III we delineate the various possibilities for the thermal history of EWSB in the model and introduce two representative scenarios. Section IV gives a detailed discussion of the conditions needed for successful EWBG, also known as a SFOEWPT: sufficiently rapid bubble nucleation and sufficiently efficient baryon number preservation inside the broken phase bubbles. In Section V we review the methods for performing a GI analysis and RG improvement. Section VI contains an extensive numerical study of the two representative EWSB scenarios given in Sec. III. In Secs. VII and VIII we then apply the constraints from DM phenomenology. We conclude in Sec. IX.

\section{MODEL}

Consider the SM extended with a complex gauge-singlet scalar field (cxSM). Following Ref. [31], we analyze a simplified version of the Higgs potential,

$$
\begin{aligned}
V_{0}(H, \mathbb{S})= & \frac{m^{2}}{2} H^{\dagger} H+\frac{\lambda}{4}\left(H^{\dagger} H\right)^{2}+\frac{\delta_{2}}{2} H^{\dagger} H|\mathbb{S}|^{2}+\frac{b_{2}}{2}|\mathbb{S}|^{2} \\
& +\frac{d_{2}}{4}|\mathbb{S}|^{4}+\left[a_{1} \mathbb{S}+\frac{b_{1}}{4} \mathbb{S}^{2}+\text { H.c. }\right],
\end{aligned}
$$


where $\mathbb{S}$ is the complex singlet scalar. The terms in the first line on the right-hand side (rhs) of Eq. (2) are invariant under the global $\mathrm{U}(1)$ transformation $\mathbb{S} \rightarrow \exp (i \theta) \mathbb{S}$. The remaining terms on the rhs explicitly break the global U(1) symmetry. The coefficients $a_{1}$ and $b_{1}$ are generally complex. While one of them can be made real through the field redefinition of $\mathbb{S}$, in general $\operatorname{Im}\left(b_{1}^{*} a_{1}^{2}\right)$ is nonvanishing. The existence of a viable DM candidate requires that one set the rephasing invariant $\operatorname{Arg}\left(b_{1}^{*} a_{1}^{2}\right)=0$. Note that one may include additional renormalizable operators that break the global U(1) [31]. But for simplicity, we have only included a minimal subset that closes under renormalization. The study of Ref. [33] includes a subset of these additional operators.

In the limit of vanishing $a_{1}$ and $b_{1}$, the occurrence of a nonzero vacuum expectation value $(\mathrm{VEV})$ of $\mathbb{S},\langle\mathbb{S}\rangle=v_{S}$, breaks the global $\mathrm{U}(1)$, giving rise to a massless NambuGoldstone mode, $A$. The $a_{1}$ and $b_{1}$ terms induce a nonzero $A$ mass without sacrificing its stability, thereby making it a viable cold DM candidate. In the limit of vanishing $a_{1}$, $V(H, \mathbb{S})$ also possesses a $\mathbb{Z}_{2}$ symmetry that would be spontaneously broken by a nonvanishing $v_{S}$. The explicit $\mathbb{Z}_{2}$-breaking $a_{1}$ term thus eliminates any potentially problematic domain walls (see, e.g., [37] and references therein). After EWSB, where $\left\langle H^{0}\right\rangle=v$, the $\mathrm{U}(1)$-symmetric $\delta_{2}$ term leads to mixing between the SM Higgs boson and the remaining degree of freedom in $\mathbb{S}$, leading to the two mass eigenstates $H_{1,2}$.

For concreteness, we choose the following representations for the scalar fields after spontaneous symmetry breaking:

$$
\begin{gathered}
H(x)=\left(\begin{array}{c}
G^{+}(x) \\
\frac{1}{\sqrt{2}}\left(v_{0}+h(x)+i G^{0}(x)\right)
\end{array}\right), \\
\mathbb{S}(x)=\frac{1}{\sqrt{2}}\left(v_{S 0}+S(x)+i A(x)\right),
\end{gathered}
$$

where $v_{0}(\simeq 246 \mathrm{GeV})$ and $v_{S 0}$ are the VEVs at zero temperature, and $G^{0, \pm}$ are the Nambu-Goldstone bosons. For a recent analysis of the Higgs phenomenology of this model and a comparison with the next-to-minimal supersymmetric standard model, see Ref. [38] (for a vacuum stability analysis at the two-loop level, see also Ref. [39]). In the current investigation, all the complex phases that can mix $S(x)$ with $A(x)$ are assumed to be 0 , and $A(x)$ is assumed to be the DM candidate.

The tadpole conditions of $V_{0}$ for $h$ and $S$ are respectively

$$
\begin{gathered}
\left\langle\frac{\partial V_{0}}{\partial h}\right\rangle=v_{0}\left[\frac{m^{2}}{2}+\frac{\lambda}{4} v_{0}^{2}+\frac{\delta_{2}}{4} v_{S 0}^{2}\right]=0 \\
\left\langle\frac{\partial V_{0}}{\partial S}\right\rangle=v_{S 0}\left[\frac{b_{2}}{2}+\frac{\delta_{2}}{4} v_{0}^{2}+\frac{d_{2}}{4} v_{S 0}^{2}+\sqrt{2} \frac{a_{1}}{v_{S 0}}+\frac{b_{1}}{2}\right]=0,
\end{gathered}
$$

where the symbol $\langle\cdots\rangle$ denotes that the fluctuating fields are taken to be 0 .

For the $v_{S 0} \neq 0$ case, after imposing Eqs. (5) and (6), the mass matrix of the $C P$-even bosons takes the form

$$
\mathcal{M}_{S}^{2}=\left(\begin{array}{cc}
\frac{\lambda}{2} v_{0}^{2} & \frac{\delta_{2}}{2} v_{0} v_{S 0} \\
\frac{\delta_{2}}{2} v_{0} v_{S 0} & \frac{d_{2}}{2} v_{S 0}^{2}-\frac{\sqrt{2} a_{1}}{v_{S 0}}
\end{array}\right),
$$

which can be diagonalized by an orthogonal matrix $O$,

$$
O^{T} \mathcal{M}_{S}^{2} O=\left(\begin{array}{cc}
m_{H_{1}}^{2} & 0 \\
0 & m_{H_{2}}^{2}
\end{array}\right), \quad O(\alpha)=\left(\begin{array}{cc}
c_{\alpha} & -s_{\alpha} \\
s_{\alpha} & c_{\alpha}
\end{array}\right),
$$

where $\alpha$ is the mixing angle such that $(h, S)^{T}=$ $O(\alpha)\left(H_{1}, H_{2}\right)^{T}$. In our study, we consider a case in which $H_{1}\left(H_{2}\right)$ is the doublet (singlet) -like Higgs boson by focusing on the range, $-\pi / 4 \leq \alpha \leq \pi / 4$.

In our study, we fix $m_{H_{1}}=125 \mathrm{GeV}$. Under this assumption, $\lambda\left(d_{2}\right)$ for a nonzero $\alpha$ is always greater (smaller) than that in the $\alpha=0$ case. Similarly, the $C P$ odd scalar mass is given by

$$
m_{A}^{2}=-\frac{\sqrt{2} a_{1}}{v_{S 0}}-b_{1} .
$$

Using Eqs. (5)-(9), one can trade off some of the original parameters with physically relevant quantities. Explicitly, we take the following as the input parameters: $v_{0}, v_{S 0}$, $m_{H_{1}}(=125 \mathrm{GeV}), m_{H_{2}}, \alpha, m_{A}$, and $a_{1}$.

For the $v_{S 0}=0$ and $a_{1}=0$, on the other hand, one has

$$
\begin{gathered}
m_{H_{1}}^{2}=\frac{\lambda}{2} v_{0}^{2}, \quad m_{H_{2}}^{2}=\frac{b_{1}+b_{2}}{2}+\frac{\delta_{2}}{4} v_{0}^{2}, \\
m_{A}^{2}=\frac{-b_{1}+b_{2}}{2}+\frac{\delta_{2}}{4} v_{0}^{2} .
\end{gathered}
$$

The input parameters in this case are $v_{0}, m_{H_{1}}(=125 \mathrm{GeV})$, $m_{H_{2}}, m_{A}, \delta_{2}$, and $d_{2}$.

\section{THERMAL HISTORY}

The behavior of the potential $V(H, \mathbb{S})$ at nonzero temperature, $T$, can lead to different patterns of symmetry breaking, depending on the choice of model parameters. To gain some intuition for various possibilities, we consider the impact of the thermal mass contributions that are responsible for symmetry restoration at high $T$. For simplicity, we begin by setting $a_{1}=0$. We return to a discussion of the full set of thermal loop contributions later. The "high-temperature effective potential" in this case is given by

$V^{\mathrm{HT}}\left(\varphi, \varphi_{S} ; T\right)=V_{0}\left(\varphi, \varphi_{S}\right)+\frac{1}{2}\left(\Sigma_{H} \varphi^{2}+\frac{1}{2} \Sigma_{S} \varphi_{S}^{2}\right) T^{2}$, 
where $\varphi$ and $\varphi_{S}$ denote the neutral doublet and singlet background fields, respectively, and

$\Sigma_{H}=\frac{\lambda}{8}+\frac{\delta_{2}}{24}+\frac{3 g_{2}^{2}+g_{1}^{2}}{16}+\frac{y_{t}^{2}}{4}, \quad \Sigma_{S}=\frac{\delta_{2}+d_{2}}{12}$,

where $g_{1}$ and $g_{2}$ denote, respectively, the $\mathrm{U}(1)_{Y}$ and $\mathrm{SU}(2)_{L}$ gauge couplings.

The various possibilities for the thermal history of EWSB are illustrated in Fig. 1. The transition to the present "Higgs phase" vacuum may occur either in two steps [panels (a)-(c)] or a single step [panel (d)]. EWSB at $T=0$ requires $m^{2}<0$ but $b_{2}$ may have either sign. For $b_{2}>0$, the only relevant impact of the singlet scalars is via thermal loops. As shown in Refs. [20,40], one requires a large number of additional singlet scalars to yield a SFOEWPT in this case. Consequently, we focus on scenarios where $m^{2}<0$ and $b_{2}<0$, for which $T=0$ minima exist for both $v_{0}$ and $v_{S 0}$ nonzero.

In the limit of vanishing $a_{1}$, the only minimum of the theory at sufficiently high $T$ occurs at the origin, denoted by "O." As $T$ decreases, one generically expects that a secondary minimum at $\varphi_{S} \equiv \bar{v}_{S}^{\mathrm{A}} \neq 0$ will first appear, since $\Sigma_{S}<\Sigma_{H}$. At a temperature $T_{1}$, the minimum at $\bar{v}_{S}^{\mathrm{A}}$ becomes the global minimum, indicated by "A" in Fig. 1. As $T$ further decreases, an additional minimum at $(\varphi \equiv \bar{v} \neq 0$, $\varphi_{S} \equiv \bar{v}_{S}^{\mathrm{B}} \neq 0$ ) develops, becoming the global minimum at temperature $T_{2}<T_{1}$, corresponding to point "B" in Fig. 1. The Universe then follows a two-step symmetry-breaking

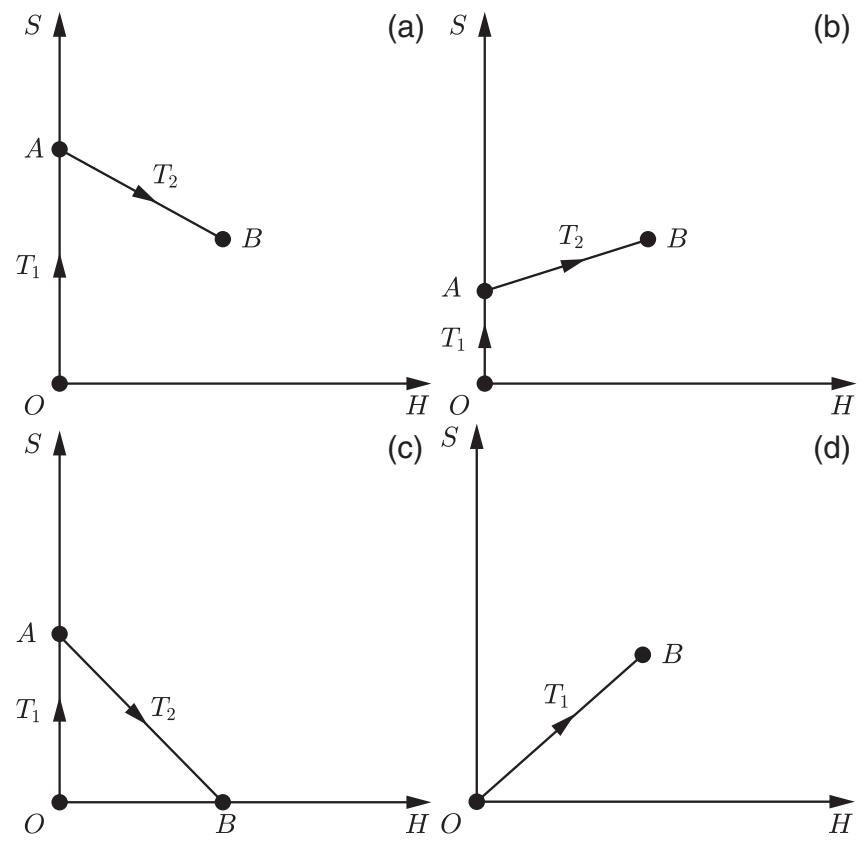

FIG. 1. Patterns of symmetry breaking at finite temperature for $a_{1}=0$. For cases (a) and (b), one has $\delta_{2}>0$ and $\delta_{2}<0$, respectively. For case (c), the singlet VEV is nonzero at $T \neq 0$ while 0 at $T=0$. For case (d), the EWPT occurs in one step. trajectory in the field space shown in Fig. 1, where one may have either $\bar{v}_{S}^{\mathrm{A}}\left(T_{2}\right)-\bar{v}_{S}^{\mathrm{B}}\left(T_{2}\right)>0$ or $<0$. We henceforth denote $T_{2}$ as the EWSB critical temperature, $T_{C}$, and the value of $\varphi$ at this temperature as $\bar{v}\left(T_{C}\right)$. After a straightforward calculation, one finds

$$
\begin{aligned}
\bar{v}\left(T_{C}\right) & \simeq \sqrt{\frac{2 \delta_{2} \bar{v}_{S}^{\mathrm{A}}\left(T_{C}\right)}{\lambda}\left(\bar{v}_{S}^{\mathrm{A}}\left(T_{C}\right)-\bar{v}_{S}^{\mathrm{B}}\left(T_{C}\right)\right)}, \\
T_{C} & \simeq \sqrt{\frac{1}{2 \Sigma_{H}}\left(-m^{2}-\frac{\left(\bar{v}_{S}^{\mathrm{A}}\left(T_{C}\right)\right)^{2}}{2} \delta_{2}\right)} .
\end{aligned}
$$

Here, the bar over $v\left(T_{C}\right)$ indicates that it has been computed using the high- $T$ effective potential given in Eq. (11). $T_{C}$ and $\bar{v}\left(T_{C}\right)$ obtained in this way are the leading-order gauge-invariant results. For positive (negative) $\delta_{2}$ one has $\bar{v}_{S}^{\mathrm{A}}\left(T_{C}\right)$ larger (smaller) than $\bar{v}_{S}^{\mathrm{B}}\left(T_{C}\right)$. In addition, for positive $\delta_{2}$, the potential has a potential barrier between the minima at A and B. In this case, the EWPT at $T=T_{C}$ is first order. Note, however, that a sufficiently large, positive $\delta_{2}$ may render B at $T=0$ metastable, since the energy difference between phase A and phase B can become negative. Therefore, there should be an upper bound on the magnitude of $\delta_{2}$, as is discussed below. For negative $\delta_{2}$, in contrast, $T_{C}$ is always raised to prevent $\bar{v}\left(T_{C}\right) / T_{C}$ from becoming sizeable. In fact, our numerical analysis (see below) does not yield a SFOEWPT for $\delta_{2}<0$.

Alternately, the EWSB may occur directly from the origin to point $\mathrm{B}$, as shown by type (d) in Fig. 1 . In the high$T$ effective theory, this transition is not first order, since $\bar{v}_{S}^{\mathrm{A}}$ is 0 , as seen from Eq. (13). However, the additional thermal corrections appearing in $V_{1}(\boldsymbol{\varphi} ; T)$ (defined below) can generate a thermal barrier that, in principle, may accommodate a first-order transition.

Using the high- $T$ potential with the thermal cubic term of $S$, one finds [31]

$$
\frac{v\left(T_{C}\right)}{T_{C}} \simeq \frac{8 E_{S}}{\lambda+2 \delta_{2} \tan ^{2} \gamma\left(T_{C}\right)+d_{2} \tan ^{4} \gamma\left(T_{C}\right)},
$$

where $\tan \gamma\left(T_{C}\right)=v_{S}\left(T_{C}\right) / v\left(T_{C}\right)$ and $E_{S}$ denotes the coefficient of the thermal cubic term of $S$. In this case, as opposed to the aforementioned two-step EWPT cases, SFOEWPT is strengthened by the negative $\delta_{2}$ term. However, it should be emphasized that $E_{S}$ would be highly suppressed if the singlet Higgs mass is dominated by the mass parameters $b_{1}$ and $b_{2}$, as in the Higgs thermal loop.

In our numerical study below, we do not find any SFOEWPT-viable parameter choices for this onestep transition. In addition, inclusion of additional U(1)breaking cubic operators, such as $H^{\dagger} H \mathbb{S}$ and/or $\mathbb{S}^{3}$ may also allow for a SFOEWPT $[18,33]$ as they contribute to the numerator in Eq. (15). 
The situation can be more complex when taking $a_{1} \neq 0$ and including the remaining zero-temperature and thermal loop effects encoded in the one-loop effective potential,

$$
V_{\text {eff }}(\boldsymbol{\varphi} ; T)=V_{0}(\boldsymbol{\varphi})+V_{1}(\boldsymbol{\varphi} ; T),
$$

where $\boldsymbol{\varphi}=\left(\varphi, \varphi_{S}\right)$,

$$
V_{1}(\boldsymbol{\varphi} ; T)=\sum_{j} n_{j}\left[V_{\mathrm{CW}}\left(\bar{m}_{j}^{2}\right)+\frac{T^{4}}{2 \pi^{2}} I_{B, F}\left(\frac{\bar{m}_{j}^{2}}{T^{2}}\right)\right],
$$

$n_{j}$ counts the degrees of freedom for particle species $j$, and $\bar{m}_{j}$ are $\boldsymbol{\varphi}$-dependent masses. The Coleman-Weinberg potential $V_{\mathrm{CW}}$ and $I_{B, F}\left(a^{2}\right)$ are respectively given by [41,42]

$$
\begin{gathered}
V_{\mathrm{CW}}\left(m^{2}\right)=\frac{m^{4}}{64 \pi^{2}}\left(\ln \frac{m^{2}}{\mu^{2}}-c\right), \\
I_{B, F}\left(a^{2}\right)=\int_{0}^{\infty} d x x^{2} \ln \left(1 \mp e^{-\sqrt{x^{2}+a^{2}}}\right),
\end{gathered}
$$

where $c=3 / 2$ for scalars and fermions and $5 / 6$ for gauge bosons, and $\mu$ is the renormalization scale.

For $a_{1} \neq 0$, the high- $T$ minimum no longer lies at the origin but is shifted by $-a_{1}$ along the $\varphi_{S}$ direction to $\mathrm{O}^{\prime}$, as illustrated in Fig. 2. The transition from $\mathrm{O}^{\prime} \rightarrow \mathrm{A}$ is a continuous process as the temperature drops.

In what follows, we exclusively explore illustrative two scenarios: Type-(a) EWPT with $a_{1} \neq 0$ (corresponding to Fig. 2) and type-(c) EWPT with $a_{1}=0$. More explicitly, we consider two scenarios.

S1: $m_{H_{2}}=230 \mathrm{GeV}, v_{S 0}=40 \mathrm{GeV}, a_{1}=-(110 \mathrm{GeV})^{3}$, S2: $m_{H_{2}}=m_{A}, v_{S 0}=0 \mathrm{GeV}, \delta_{2}=0.55, d_{2}=0.5$, and $m_{A}=m_{H_{1}} / 2=62.5 \mathrm{GeV}$ in both cases. ${ }^{2}$ In Fig. 3 , the evolution of the VEVs with temperature is plotted for S1 (left) and S2 (right). For the former, there is no $\mathrm{O} \rightarrow \mathrm{A}$ transition, as shown in Fig. 2, and we find that the A $\rightarrow$ B transition is first order since $\bar{v}$ has a discontinuity at $T_{2}=T_{C}=90.4 \mathrm{GeV}$ with $\bar{v}\left(T_{C}\right)=158.2 \mathrm{GeV}$. For the latter, on the other hand, one can see that the $\mathrm{O} \rightarrow \mathrm{A}$ transition is second order, with $T_{1}=224.6 \mathrm{GeV}$, while the $\mathrm{A} \rightarrow \mathrm{B}$ transition is first order, with $T_{2}=T_{C}=$ $99.8 \mathrm{GeV}, \quad \bar{v}\left(T_{C}\right)=167.0 \mathrm{GeV}, \quad \bar{v}_{S}^{\mathrm{B}}\left(T_{C}\right)=0, \quad$ and $\bar{v}_{S}^{\mathrm{A}}\left(T_{C}\right)=168.4 \mathrm{GeV}$.

Figure 4 shows contours of the high- $T$ effective potential at $T=250 \mathrm{GeV}$ (upper left), $T_{C}+5 \mathrm{GeV}$ (upper right), $T_{C}$ (lower left), and $0 \mathrm{GeV}$ (lower right) in the case of S2. The minima of the potential are indicated by the large black dots. One can see that at $T_{C}$ there exists a barrier between the pure singlet VEV [point A of Fig. 1(c)] and the pure

\footnotetext{
${ }^{2}$ We take $m_{A}$ as a variable parameter when discussing the DM phenomenology in Sec. VII. Furthermore, $H_{2}(=S)$ is also the $\mathrm{DM}$ candidate in $\mathrm{S} 2$.
}

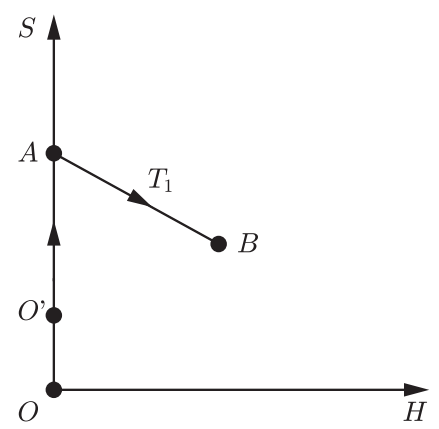

FIG. 2. Symmetry breaking at finite temperature for $a_{1} \neq 0$. In this case the $\mathrm{O} \rightarrow \mathrm{A}$ transition is absent, and the initially nonzero $\bar{v}_{S}^{\mathrm{A}}(T)$ smoothly increases until the temperature reaches $T_{1}$ where EWPT happens.

doublet VEV [point B of Fig. 1(c)]. At $T=0$, the only minimum occurs along the doublet direction whereas the extremum along the singlet direction is a saddle point.

\section{BARYOGENESIS}

EWBG requires that the transition to the EWSB vacuum $\mathrm{B}$ be strongly first order, associated with both bubble nucleation and quenching of the EW sphalerons inside the bubbles. The nucleation rate, $\Gamma_{N}$, is governed by the threedimensional effective action, $S_{3}$, which is typically computed using the bounce solution in the presence of $V_{\text {eff }}(\boldsymbol{\varphi} ; T)$ at the one-loop order. In this case, the gauge dependence must be treated with some care. In this paper, we use the high- $T$ effective potential, Eq. (11), for the evaluation of $\Gamma_{N}$ as a first step toward more complete analyses. For the regions of parameter space explored in this study, the tree-level $T=0$ potential contains a barrier between the Higgs phase vacuum and the electroweak symmetric vacuum. Consequently, we need not consider scenarios with a thermally induced barrier that introduces problematic gauge dependence. ${ }^{3}$

The nucleation temperature, $T_{N}$, is defined as a temperature that satisfies $\Gamma_{N}\left(T_{N}\right) / H^{3}\left(T_{N}\right)=H\left(T_{N}\right)$ with $H(T)$ being a Hubble constant [43]. ${ }^{4}$ With an approximation of $\Gamma_{N}$ discussed in Ref. [43], the above condition may be cast into the form

$$
\begin{aligned}
\frac{S_{3}\left(T_{N}\right)}{T_{N}}-\frac{3}{2} \ln \left(\frac{S_{3}\left(T_{N}\right)}{T_{N}}\right)= & 152.59-2 \ln g_{*}\left(T_{N}\right) \\
& -4 \ln \left(\frac{T_{N}}{100 \mathrm{GeV}}\right),
\end{aligned}
$$

\footnotetext{
${ }^{3} \mathrm{We}$ also observe that for scenarios considered here, use of an $\hbar$-expansion to obtain a gauge-invariant $S_{3}$ is, in principle, possible.

${ }^{4}$ Since the bubble velocity is less than the speed of light, a single nucleated bubble is not able to convert the entire region of the Universe to the broken phase. Thus, $T_{N}$ defined here merely represents an upper bound for the onset of the EWPT.
} 

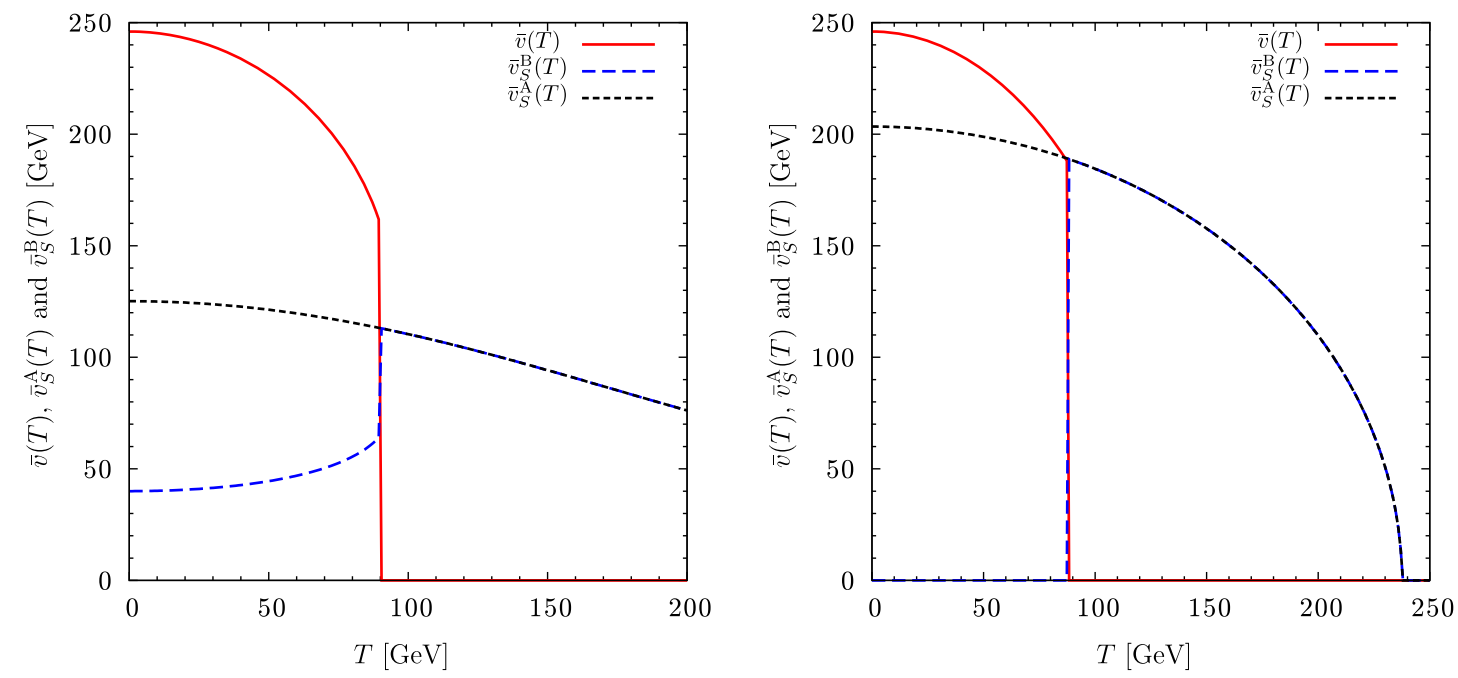

FIG. 3. Evolution of VEVs as a function of $T$ using the high- $T$ effective potential in type-(a) EWPT with $a_{1} \neq 0$ (left) and type-(c) EWPT with $a_{1}=0$ (right). For the former, the $A \rightarrow B$ transition is first order, with $T_{2}=T_{C}=90.4 \mathrm{GeV}$ and $\bar{v}\left(T_{C}\right)=158.2 \mathrm{GeV}$. For the latter, the $O \rightarrow A$ transition is second order while the $A \rightarrow B$ transition is first order. It is found that $T_{1}=224.6 \mathrm{GeV}$, $T_{2}=T_{C}=99.8 \mathrm{GeV}$, and $\bar{v}\left(T_{C}\right)=167.0 \mathrm{GeV}$.

where $g_{*}(T)$ is the number of relativistic degrees of freedom in the plasma. Roughly speaking, Eq. (20) implies that $S_{3}\left(T_{N}\right) / T_{N} \simeq 150$ is needed for the development of EWPT [43]. As we demonstrate below, choosing parameters to decrease the broken phase sphaleron rate (thereby suppressing baryon number washout) also leads to a larger $S_{3}\left(T_{N}\right) / T_{N}$. Eventually, one cannot fulfil the condition of Eq. (20).

Assuming a sufficient nucleation rate, preservation of the baryon asymmetry inside the bubbles imposes a requirement on the EW sphaleron transition rate,

$$
\Gamma_{\mathrm{sph}}=A_{\mathrm{sph}}(T) \exp \left[-E_{\mathrm{sph}}(T) / T\right]
$$

where $E_{\text {sph }}$ is the sphaleron energy, $A_{\text {sph }}$ is a temperaturedependent prefactor, and $T \lesssim T_{N}$ with $T_{N}$ being the nucleation temperature that typically lies just below $T_{C}$. The effect of baryon number washout inside the bubbles is characterized by the washout factor

$$
S=\frac{n_{B}\left(\Delta t_{\mathrm{EW}}\right)}{n_{B}(0)}
$$

where $n_{B}(t)$ is the baryon number density at a time $t$ after the onset of the transition, $n_{B}(0)$ is the initial baryon number density, and $\Delta t_{\mathrm{EW}}$ is the duration of the EWPT. Requiring that $S>\exp (-X)$, one obtains the baryon number preservation criterion (BNPC) [35],

$$
\begin{aligned}
& \frac{E_{\mathrm{sph}}\left(T_{C}\right)}{T_{C}}-7 \ln \frac{\bar{v}\left(T_{C}\right)}{T_{C}}>-\ln X-\ln \left(\frac{\Delta t_{\mathrm{EW}}}{t_{H}}\right) \\
& \quad+\ln \mathcal{Q F}+\ln \kappa,
\end{aligned}
$$

where $t_{H}$ is the Hubble time, $\kappa$ is the fluctuation determinant about the classical sphaleron solution, and $\mathcal{Q}$ and $\mathcal{F}$ encode the effects of rotational and translational zero modes as well as the unstable mode about the sphaleron. ${ }^{5}$

It is convenient to express $E_{\text {sph }}$ in terms of an energy scale $\Omega(T)$ associated with the EWSB that is typically of order $T_{C}$. To this end, we write

$$
E_{\mathrm{sph}}(T)=\frac{4 \pi \Omega(T)}{g_{2}} \mathcal{E}(T) .
$$

When the only scalar fields in the theory are $\mathrm{SU}(2)_{L}$ doublets, the natural choice for $\Omega(T)$ is $\bar{v}(T)$. For the cxSM, there exists a second possibility:

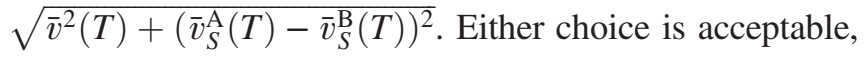
as the BNPC depends on $E_{\text {sph }}$ and the difference in the choice of $\Omega(T)$ is compensated by the corresponding convention for $\mathcal{E}(T)$. Here we follow Refs. [19,44] where it is argued that $\Omega(T)=\bar{v}(T)$ encapsulates the primary $T$-dependence of the sphaleron energy. Nevertheless, we find that the residual $T$-dependence of $\mathcal{E}$ can be nonnegligible in some cases. The detailed calculation of $E_{\text {sph }}(T)$ is given in the Appendix.

From these considerations, one obtains from the BNPC (23) a requirement on the ratio $\bar{v}\left(T_{C}\right) / T_{C}$,

$$
\frac{\bar{v}\left(T_{C}\right)}{T_{C}} \gtrsim \zeta_{\mathrm{sph}}\left(T_{C}\right)
$$

\footnotetext{
${ }^{5}$ The quantity $X$ parametrizes the degree to which the initial baryon asymmetry may be diluted by sphaleron processes. Its value depends on the initial value of the asymmetry obtained from a computation of the $\mathrm{CP}$-violating transport dynamics in a given model.
} 

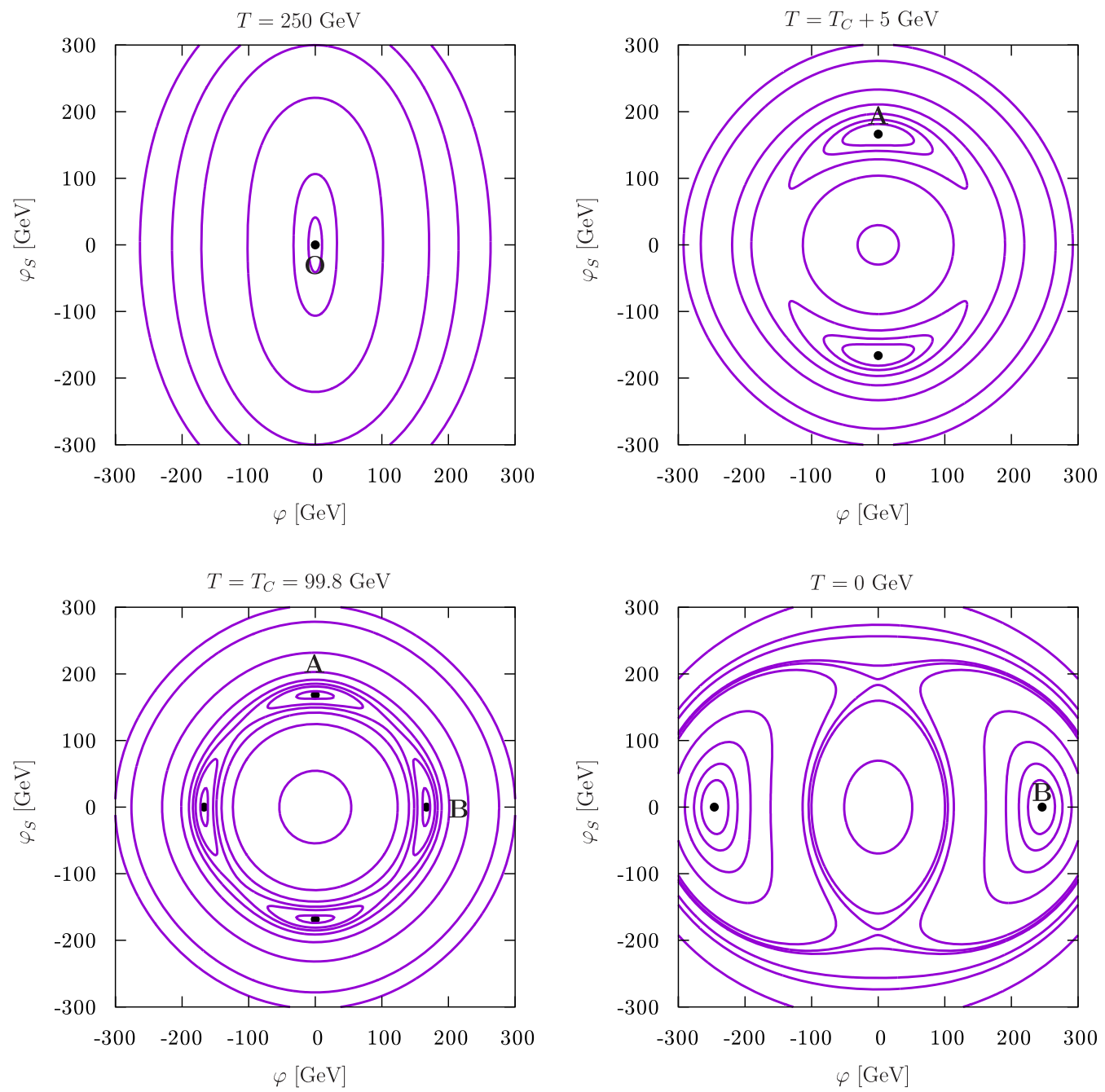

FIG. 4. Contours of high- $T$ effective potential at $T=250 \mathrm{GeV}$ (upper left), $T_{C}+5 \mathrm{GeV}$ (upper right), $T_{C}$ (lower left), and $0 \mathrm{GeV}$ (Lower Right), where $T_{C}=99.8 \mathrm{GeV}, \bar{v}\left(T_{C}\right)=167.0 \mathrm{GeV}, \bar{v}_{S}^{\mathrm{B}}\left(T_{C}\right)=0 \mathrm{GeV}, \bar{v}_{S}^{\mathrm{A}}\left(T_{C}\right)=168.4 \mathrm{GeV}$ in the case of S2. The black dots denote the minima of the potential.

In the literature, one often finds this requirement quoted as $v\left(T_{C}\right) / T_{C} \gtrsim 1$, where $v\left(T_{C}\right)$ is computed using the full one-loop effective potential $V_{\text {eff }}$ rather than $V^{\mathrm{HT}}$ [45-48]. As discussed in Ref. [35], this procedure, as well as the conventional method for computing $T_{C}$, introduces an unphysical gauge dependence. In what follows, we perform a gauge-invariant computation. We also address the impact of the $\mu$-dependence by implementing a RG-improved analysis. These and other theoretical issues associated with the BNPC and $\Gamma_{N}$ are discussed below.

\section{GAUGE-INVARIANT METHOD BEYOND THE LEADING ORDER}

Here, we delineate the gauge-invariant treatment for the EWPT and sphaleron rate. Determination of $T_{C}$ and $\bar{v}_{C}$ using the high- $T$ effective potential is obviously gauge independent. Beyond this order, however, the potential barrier inherently depends on the gauge fixing parameter, which may lead to the gauge-dependent $T_{C}$ and $v_{C}$ as in the ordinary method. Nevertheless, the gauge-invariant $T_{C}$ can still be obtained by use of a method advocated in Ref. [35] (Patel-Ramsey-Musolf (PRM) scheme). Here we outline the method briefly.

The Nielsen-Fukuda-Kugo identity [49,50] states that energies at station points of the effective potential are free from the gauge fixing parameter, which is described by

$$
\frac{\partial V_{\mathrm{eff}}(\varphi)}{\partial \xi}=-C(\varphi, \xi) \frac{\partial V_{\mathrm{eff}}(\varphi)}{\partial \varphi}
$$

where $C(\varphi, \xi)$ denotes some functional (for the explicit form, see Ref. [49]). We determine $T_{C}$ and $v\left(T_{C}\right)$ in such a way that the above identity is satisfied order by order in the perturbative expansion. Let us expand $V_{\text {eff }}$ and $C$ in powers of $\hbar$ as 


$$
\begin{array}{r}
V_{\mathrm{eff}}(\varphi)=V_{0}(\varphi)+\hbar V_{1}(\varphi)+\hbar^{2} V_{2}(\varphi)+\cdots, \\
C(\varphi, \xi)=c_{0}+\hbar c_{1}(\varphi)+\hbar^{2} c_{2}(\varphi)+\cdots .
\end{array}
$$

For example, the identity to $\mathcal{O}(\hbar)$ is found to be

$$
\frac{\partial V_{1}}{\partial \xi}=-c_{1} \frac{\partial V_{0}}{\partial \varphi}
$$

Note that the $\xi$ dependence of $V_{1}$ drops out at the points where the tree-level potential is extremized, which differs from the extremum of $V_{1}$.

Correspondingly, $T_{C}$ to $\mathcal{O}(\hbar)$ is determined by the following degeneracy condition,

$$
V_{0}\left(\boldsymbol{v}_{0}^{\mathrm{A}}\right)+V_{1}\left(\boldsymbol{v}_{0}^{\mathrm{A}} ; T_{C}\right)=V_{0}\left(\boldsymbol{v}_{0}^{\mathrm{B}}\right)+V_{1}\left(\boldsymbol{v}_{0}^{\mathrm{B}} ; T_{C}\right),
$$

where $\boldsymbol{v}_{0}^{\mathrm{A}}$ denote the VEVs of phase A while $\boldsymbol{v}_{0}^{\mathrm{B}}$ those of phase $\mathrm{B}$ as defined above.

On the other hand, $\bar{v}\left(T_{C}\right)$ in the PRM scheme is determined by the high temperature potential $V^{\mathrm{HT}}$ given in Eq. (11), which is manifestly gauge invariant.

Though the so-called ring diagrams can also be implemented in a gauge-invariant manner, knowledge of $V_{2}$ is indispensable for the consistency of the calculation. Since $V_{2}$ in this model is not available to date, we confine ourselves to the $\mathcal{O}(\hbar)$ calculation in this paper.

The appearance of the renormalization scale $\mu$ in $V_{\mathrm{CW}}$ in Eq. (17) can lead to a significant $\mu$-dependence for $T_{C}$. To alleviate this dependence, we replace $V_{0}(\boldsymbol{\varphi})$ appearing in the degeneracy condition (30) by the RG-improved potential $\tilde{V}_{0}(\boldsymbol{\varphi})$. More explicitly, we replace all the parameters in $V_{0}$ with the running ones

$$
\begin{aligned}
\tilde{V}_{0}\left(\varphi, \varphi_{S}\right)= & \frac{m^{2}\left(\mu^{2}\right)}{4} \varphi^{2}+\frac{\lambda\left(\mu^{2}\right)}{16} \varphi^{4}+\frac{\delta_{2}\left(\mu^{2}\right)}{8} \varphi^{2} \varphi_{S}^{2} \\
& +\frac{b_{2}\left(\mu^{2}\right)}{4} \varphi_{S}^{2}+\frac{d_{2}\left(\mu^{2}\right)}{16} \varphi_{S}^{4}+\sqrt{2} a_{1}\left(\mu^{2}\right) \varphi_{S} \\
& +\frac{b_{1}\left(\mu^{2}\right)}{4} \varphi_{S}^{2} .
\end{aligned}
$$

Here, we use the one-loop $\beta$ functions [32] to evaluate the running parameters, and the RG effects on $\varphi$ and $\varphi_{S}$ are ignored as they are negligible. Note that the other parameters appearing in Eq. (30) remain unchanged in order not to spoil the gauge independence to this order. Similarly, $V_{0}(\boldsymbol{\varphi})$ in Eq. (11) remains as is since the renormalization scale does not enter the high- $T$ effective potential. In what follows, we numerically demonstrate that this procedure eliminates the otherwise problematic $\mu$-dependence while maintaining gauge invariance.

\section{NUMERICAL ANALYSIS}

In this section, we perform a numerical analysis taking theoretical and experimental constraints into account. The bounded-from-below conditions for the scalar potential are imposed as

$$
\lambda>0, \quad d_{2}>0, \quad-\sqrt{\lambda d_{2}}<\delta_{2},
$$

where the last condition is only applied to the $\delta_{2}<0$ case. Furthermore, the absolute values of the quartic couplings are restricted to be less than $4 \pi$ as a simple criterion of perturbativity (for more detailed analysis and some subtleties, see Ref. [32]).

The quantities $m_{H_{2}}$ and $\alpha$ are constrained by direct searches for a heavy Higgs boson at the LHC, measurements of Higgs signal strengths [25,39], and electroweak precision observables. For example, utilizing the two gauge boson decay modes, $m_{H_{2}}$ is bounded as a function of $\sin ^{2} \alpha$ with $\mathcal{B}_{\text {new }}=0.0,0.2$ and 0.5 , where $\mathcal{B}_{\text {new }}$ denotes the non-SM contribution to decay width [51]. For the mixing angle, it is found that $\cos \alpha \gtrsim 0.8$ for $m_{\mathrm{H}_{2}} \gtrsim 250 \mathrm{GeV}$ to be consistent with the EW observables within the $3 \sigma$ level [39].

\section{A. S1 case}

Recall that scenario S1 follows the two-step history of Fig. 2 with the high- $T$ minimum lying away from the origin due to the nonvanishing $a_{1}$. Figure 5 shows the $\mu$ dependence of $T_{C}$ and the corresponding $\bar{v}\left(T_{C}\right)$ with and without the RG improvement. The input parameters are the same as in the left plot of Fig 3. The solid curves represent the former and the dashed ones the latter. One can see that $T_{C}$ has some renormalization scale dependence before the

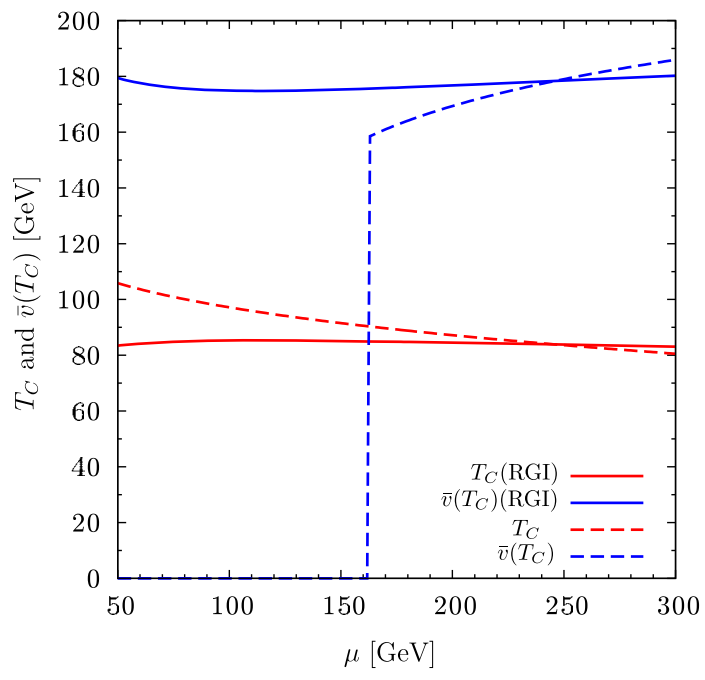

FIG. 5. Renormalization scale dependence of $T_{C}$ and $\bar{v}_{C}$ in S1. The dashed curves are calculated based on the original PRM scheme to $\mathcal{O}(\hbar)$ while the solid ones are the RG-improved version. 


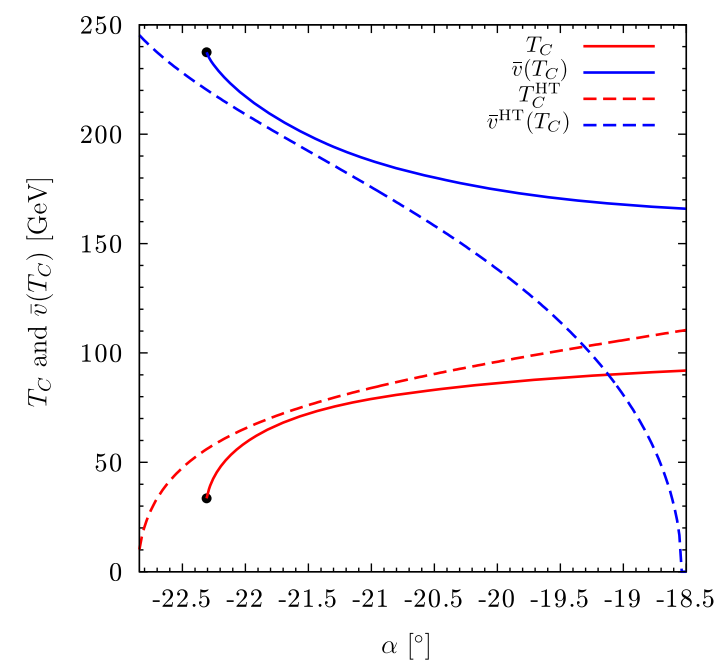

FIG. 6. $\quad T_{C}$ and $\bar{v}\left(T_{C}\right)$ as functions of $\alpha$ in S1. The solid curves are obtained by the PRM scheme with RG improvement while the dashed ones are obtained by the high- $T$ effective potential in Eq. (11).

RG improvement. This results in uncertainties in the determination of $\bar{v}\left(T_{C}\right)$, which is shown in the plot to vary from 0 to non- 0 . This makes it difficult to reach a conclusion whether or not the EWPT is of first order. After the RG improvement, however, the renormalization scale dependence is substantially alleviated, and the EWPT is seen to be strongly first order.

As discussed above, since the mixing angle $\alpha$ is one of the most important parameters for both LHC phenomenology and for a SFOEWPT, we first quantify the $\alpha$-dependence of $T_{C}$ and $\bar{v}$. In Fig. 6, $T_{C}$ and $\bar{v}\left(T_{C}\right)$ are plotted as functions of $\alpha$ where the solid curves correspond to the PRM scheme with RG improvement, while the dashed ones are calculated by use of the high- $T$ potential (HT calculation). One can see that $T_{C}$ decreases (more favorable for SFOEWPT) as $\alpha$ decreases in both cases. Recall that a positive, increasing $\delta_{2}$ also leads to a decreasing $T_{C}$, as seen from the analytic formula Eq. (14) (with $m^{2}<0$ ). For $\alpha \lesssim-22.8^{\circ}$, however, phase $A$ becomes the global minimum, yielding an upper bound $\delta_{2} \lesssim 2.7$ in the HT calculation. In the PRM calculation, there is an end point around $\alpha \simeq-22.3^{\circ}$ indicated by the black dots. Below this point, the vacuum energy of phase $B$ is higher than that of phase $A$, even at $T=0$. Consequently, the degeneracy condition (30) is never satisfied.

Note that $T_{C}<T_{C}^{\mathrm{HT}}$ in all the range and that the differences between the HT and PRM results become more prominent as $\alpha$ increases. Moreover, the tree-level potential barrier disappears for $\alpha \gtrsim-18.5^{\circ}$. Thus, in the HT calculation, the EWPT transition is second order in this region, and $\bar{v}$ can correspondingly be cast into the form $\sqrt{1-\left(T / T_{0}\right)^{2}}$, where $T_{0}$ is the critical temperature of the second-order EWPT. In the PRM calculation, on the other hand, a small loop-induced contribution to the potential remains, and one finds that $T_{C}<T_{0}$. Since this leads to $\bar{v}\left(T_{C}\right)>0$, one might naïvely interpret this to indicate the presence of a SFOEWPT. However, phase A in this case becomes a saddle point rather than a local minimum, and thus the region $\alpha \gtrsim-18.5^{\circ}$ is, in fact, excluded.

As emphasized in Ref. [35], the $\mathcal{O}(\hbar)$ computation of $T_{C}$ is likely an underestimate. In the SM, inclusion of higher order contributions, either in perturbation theory or lattice computations, yields a larger value of $T_{C}$. The corresponding theoretical uncertainty can be significant. Even though a quantitatively robust statement awaits a more precise analysis, we are able to identify some general trends: the realization of SFOEWPT in this model needs a negative $\alpha$ (corresponding to positive $\delta_{2}$ ) but not too large in magnitude, as shown by the range of Fig. 6 .

As discussed in Sec. IV, the actual beginning of the EWPT occurs at a temperature $T_{N}$ that is somewhat below the temperature at which the effective potential has two degenerate minima. ${ }^{6}$ If $T_{N}$ is sufficiently close to $T_{C}$, we can approximate $T_{C}$ as the transition temperature, and thus $E_{\text {sph }}\left(T_{C}\right) / T_{C} \simeq E_{\text {sph }}\left(T_{N}\right) / T_{N}$ approximately holds. It is useful to characterize the degree of supercooling by the quantity

$$
\Delta \equiv \frac{T_{C}-T_{N}}{T_{C}}
$$

It is known that $\Delta=\mathcal{O}(0.1) \%$ in the minimal supersymmetric SM (MSSM) case (see, e.g., Refs. [45,52]).

Here, we calculate $S_{3}(T)$ to find $T_{N}$ using the high- $T$ effective potential in Eq. (11). In Fig. 7, the solid curve shows $S_{3}(T) / T$ as a function of $T$ for $\alpha=-20.5^{\circ}$ in S1. The dotted line satisfies the condition in Eq. (20), from which we obtain $S_{3}\left(T_{N}\right) / T_{N}=152.01$ and $T_{N}=84.9 \mathrm{GeV}$. Since $T_{C}^{\mathrm{HT}}=90.4 \mathrm{GeV}$, one obtains $\Delta_{\mathrm{HT}}=6.1 \%$.

We also find that the supercooling becomes larger as $\alpha$ decreases, and eventually the condition of Eq. (20) cannot be fulfilled for $\alpha \lesssim-21.4^{\circ}$, rendering a more stringent lower bound on $\alpha$ than the vacuum degeneracy condition mentioned above. For the critical $\alpha=-21.4^{\circ}$, we obtain $T_{C}^{\mathrm{HT}}=78.1 \mathrm{GeV}$ and $T_{N}=47.3 \mathrm{GeV}$, leading to $\Delta_{\mathrm{HT}} \simeq 39.4 \%$.

The degree of supercooling affects the dynamics of the EWPT and the feasibility of EWBG. As discussed above, the first-order EWPT proceeds via the bubble nucleation and expansion. For EWBG to be successful, most of the region in the symmetric phase has to be converted into the broken phase via the bubble expansion rather than the nucleation since the baryon asymmetry is generated by the scatterings of the particles with the growing bubbles.

\footnotetext{
${ }^{6}$ Such a phenomenon, in which the phase transition delays its occurrence until the nucleation temperature lower than the critical temperature, is called supercooling.
} 


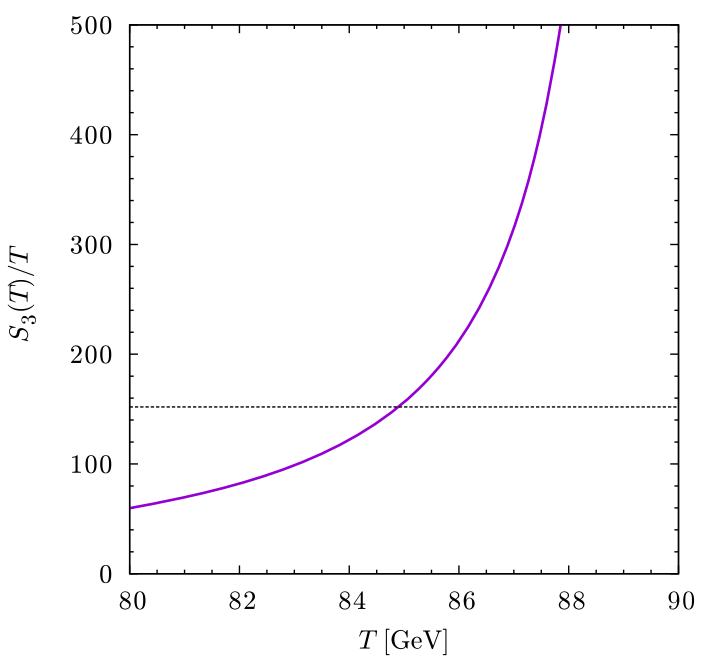

FIG. 7. $S_{3}(T) / T$ vs $T$ for $\alpha=-20.5^{\circ}$ in $\mathrm{S} 1 . S_{3}(T)$ is evaluated by use of the high- $T$ effective potential in Eq. (11). The dotted horizontal line satisfies the condition in Eq. (20). In this case, we have $T_{C}^{\mathrm{HT}}=90.4 \mathrm{GeV}$ and $T_{N}=84.9 \mathrm{GeV}$, with the latter closer to $T_{C}$ calculated in the PRM scheme.

If the supercooling $\Delta$ is large, however, the EWPT may proceed mostly via the nucleation since the nucleation rate $\Gamma_{N}$ gets enhanced, spoiling the EWBG mechanism. Prior to the discovery of the Higgs boson and top quark, these dynamics of the EWPT in the SM were studied in Ref. [53]. For $m_{h}=60 \mathrm{GeV}$ and $m_{t}=120 \mathrm{GeV}$, the supercooling is found to be around $0.25 \%$. About $10 \%$ of the symmetricphase region is converted into the broken phase when bubble nucleation completes, and the rest of the conversion results from the bubble expansion. An analogous study is needed for cxSM in order to assess the viability of EWBG.

We now turn to the final set of questions posed in Sec. I: To what extent does $\bar{v}(T)$ characterize the sphaleron energy, $E_{\mathrm{sph}}(T)$ ? To that end, we first plot in Fig. 8 the ratio $E_{\mathrm{sph}}(T) / T$ vs $T$ for $\alpha=-20.5^{\circ}$ in $\mathrm{S} 1$, where $E_{\mathrm{sph}}(T)$ is estimated based on the high- $T$ effective potential in Eq. (11). From right to left, the three dots mark the results for $E_{\mathrm{sph}}\left(T_{C}^{\mathrm{HT}}\right) / T_{C}^{\mathrm{HT}}=61.31, E_{\mathrm{sph}}\left(T_{N}\right) / T_{N}=74.23$ and $E_{\mathrm{sph}}\left(T_{C}\right) / T_{C}=78.00$ using the values of $T_{C}^{\mathrm{HT}}, T_{N}$ and $T_{C}$ given above. Recall that for this value of $\alpha$, the tree-level potential admits a barrier between the phases $\mathrm{A}$ and $\mathrm{B}$, so that the EWPT is first order, even in the HT framework. Thus, $T_{C}^{\mathrm{HT}}<T_{0}$, where $T_{0}$ is the temperature at which the gauge-invariant scale $\bar{v}(T)$ vanishes, implying a vanishing $E_{\mathrm{sph}}(T)$ in the computational framework adopted here. The end point at $T_{C}^{\mathrm{HT}}$ simply implies that of the computational approaches discussed here, the one giving the maximum $T_{C}$, and thus, the minimum (but nonvanishing) $E_{\text {sph }}\left(T_{C}\right) / T_{C}$, is the HT computation.

In Fig. 9, the dimensionless sphaleron energy $\mathcal{E}(T)$ is plotted as a function of $T$. Apparently, $\mathcal{E}(T)$ decreases as $T$ increases, showing that the temperature dependence of

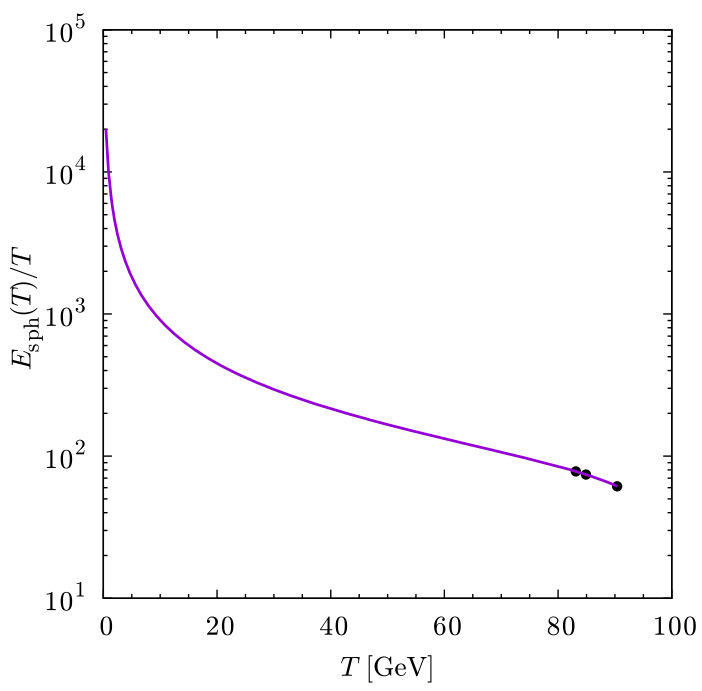

FIG. 8. $E_{\mathrm{sph}}(T) / T$ as a function of $T$, where $E_{\mathrm{sph}}(T)$ is calculated using the high- $T$ effective potential in Eq. (11). From right to left, the dots mark for $E_{\mathrm{sph}}\left(T_{C}^{\mathrm{HT}}\right) / T_{C}^{\mathrm{HT}}=61.31$, $E_{\text {sph }}\left(T_{N}\right) / T_{N}=74.23$ and $E_{\text {sph }}\left(T_{C}\right) / T_{C}=78.00$, where $T_{C}^{\mathrm{HT}}=90.4 \mathrm{GeV}, T_{N}=84.9 \mathrm{GeV}$ and $T_{C}=83.1 \mathrm{GeV}$.

$E_{\mathrm{sph}}(T)$ is not fully embodied in $\Omega(T)$, where we have taken $\Omega(T)=\bar{v}(T)$ as indicated above. We conclude that the naïve scaling formula $E_{\text {sph }}(T)=E_{\text {sph }}(0) \bar{v}(T) / v_{0}$ is no longer valid, especially when $T$ approaches $T_{C}$ (for earlier studies, see Refs. $[45,46,54])$. As in Fig. 8, the three dots correspond to $\mathcal{E}\left(T_{C}^{\mathrm{HT}}\right)=1.82, \mathcal{E}\left(T_{N}\right)=1.86$ and $\mathcal{E}\left(T_{C}\right)=$ 1.87 from right to left. Because the deviation from a linear dependence of $E_{\mathrm{sph}}(T)$ on $\bar{v}(T)$ is particularly pronounced in the vicinity of the critical temperature, one should apply a fair degree of caution before drawing sharp conclusions about the viability of EWBG based on a

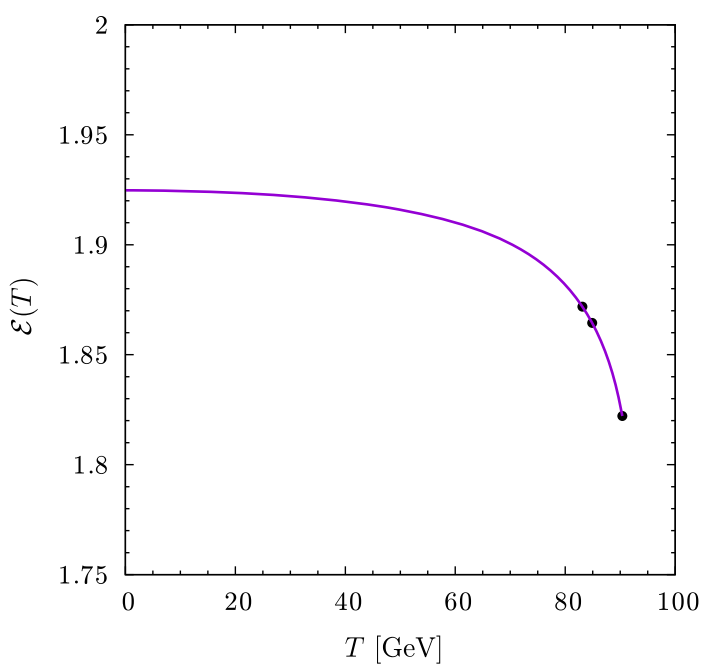

FIG. 9. $\mathcal{E}(T)=g_{2} E_{\mathrm{sph}}(T) /(4 \pi \bar{v})$ as a function of $T$. The three dots correspond to $\mathcal{E}\left(T_{C}^{\mathrm{HT}}\right)=1.82, \mathcal{E}\left(T_{N}\right)=1.86$ and $\mathcal{E}\left(T_{C}\right)=$ 1.87 from right to left. 
one-loop perturbative treatment of the EWPT. As we have emphasized earlier, inclusion of higher order contributions can lead to significant variations in $T_{C}$, implying corresponding variations in the $E_{\mathrm{sph}}(T) / T$ and, thus, the efficacy of baryon number preservation in the broken phase.

\section{B. $\mathbf{S} 2$ case}

This scenario corresponds to the thermal history of Fig. 1(c). Because the singlet VEV vanishes at $T=0$, one has $\alpha=0$. Moreover, there is no constraint among the parameters $b_{1}, b_{2}, \delta_{2}$ and $d_{2}$ from the tadpole condition of Eq. (6), in contrast to the $\mathrm{S} 1$ case. Consequently, it is most instructive to consider the dependence of EWPT properties on the portal coupling $\delta_{2}$. For purposes of the following discussion, we also note that a necessary condition for the existence of the nontrivial vacuum phase $\mathrm{A}$ in $\varphi_{S}$ is

$$
\left[\bar{v}_{S}^{\mathrm{A}}(T)\right]^{2}=-\frac{2}{d_{2}}\left(b_{1}+b_{2}+2 \Sigma_{S} T^{2}\right)>0,
$$

which implies that $b_{1}+b_{2}$ must be negative as long as $\Sigma_{S}$ is positive. While necessary, this condition is not sufficient since a saddle point is also possible.

Figure 10 shows the dependence of $T_{C}$ and $\bar{v}\left(T_{C}\right)$ on $\delta_{2}$. The line and color styles are the same as in Fig. 6. Below the left end point at $\delta_{2} \simeq 0.51$, the potential barrier is eliminated and thus the EWPT is of second order. In the HT calculation, phase A becomes the global minimum at $T=0$ for $\delta_{2} \gtrsim 0.77$. In this case, the EWPT never occurs. In the PRM case, $T_{C}$ is lower than the HT case as explained above. Moreover, as in the case of $\mathrm{S} 1$, there is a point where $T_{C}$ cannot be defined due to the absence of vacuum

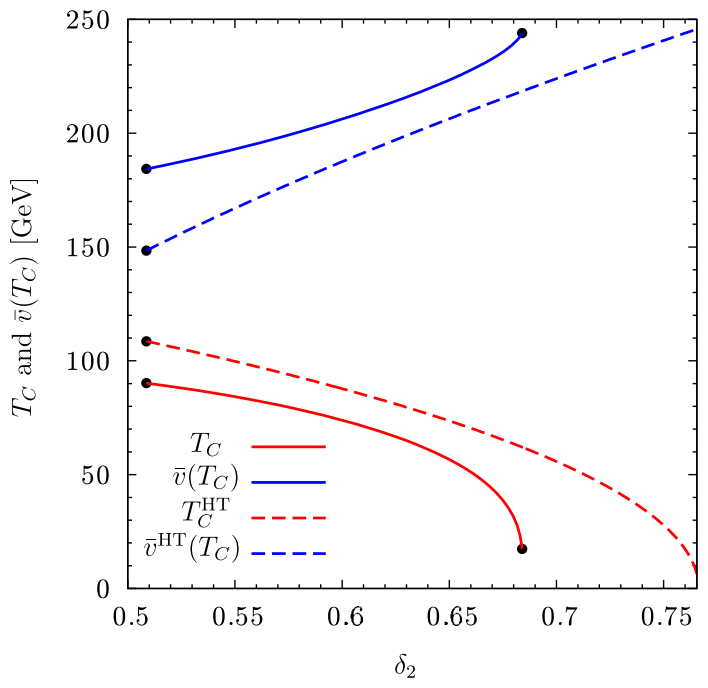

FIG. 10. $T_{C}$ and $\bar{v}\left(T_{C}\right)$ as functions of $\delta_{2}$. Below the left end point $\left(\delta_{2} \simeq 0.51\right)$ the EWPT is second order for both HT and PRM cases, while $T_{C}$ cannot be defined above the right end point $\left(\delta_{2} \simeq 0.68\right)$ in the PRM case. For $\delta_{2} \gtrsim 0.77$, phase A turns into the global minimum at $T=0$ in the HT case.
TABLE I. VEVs and sphaleron energies at different temperatures, $T_{C}, T_{C}^{\mathrm{HT}}$ and $T_{N}$, for $\delta_{2}=0.55$ in $\mathrm{S} 2$. The last two columns are calculated by use of the high- $T$ effective potential (11).

\begin{tabular}{lccc}
\hline \hline$T(\mathrm{GeV})$ & $T_{C}=84.3$ & $T_{C}^{\mathrm{HT}}=99.8$ & $T_{N}=96.6$ \\
\hline $\bar{v}(T)(\mathrm{GeV})$ & 193.0 & 167.0 & 173.5 \\
$E_{\mathrm{sph}}(T) / T$ & 84.36 & 61.67 & 66.02 \\
$\mathcal{E}(T)$ & 1.92 & 1.92 & 1.92 \\
\hline \hline
\end{tabular}

degeneracy, which corresponds to the right end point at $\delta_{2} \simeq 0.68$. Our findings show that the region that is consistent with SFOEWPT is generally more limited in the PRM case.

As in the $\mathrm{S} 1$ case, we also evaluate $S_{3}(T), E_{\mathrm{sph}}(T)$ and $\mathcal{E}$, fixing $\delta_{2}=0.55$. The results are summarized in Table I. One can see that as in the S1 case, the degree of the supercooling is $\Delta_{\mathrm{HT}} \simeq 3.2 \%$, about 1 order of magnitude larger than the typical MSSM value [45,52]. However, one distinctive feature of $\mathrm{S} 2$ is that $\mathcal{E}$ is independent of $T$, implying that $E_{\mathrm{sph}}(T)=E_{\mathrm{sph}}(0) \bar{v}(T) / v_{0}$ as in the SM. This is due to the fact that there is no singlet Higgs contribution to $E_{\text {sph }}$ since $\bar{v}_{S}=0$.

Before closing this section, we comment on the impact of the DM mass on the viability of a SFOEWPT. In both the $\mathrm{S} 1$ and $\mathrm{S} 2$ cases, the $T=0$ vacuum energy of phase $B$ increases as the DM mass increases and surpasses the vacuum energy of phase $A$ at some particular value. Hence, no transition to phase $\mathrm{B}$ occurs and $T_{C}$ cannot be defined in the PRM calculation. As is discussed in the next section, a relatively large DM mass is generally required to obtain the correct DM relic abundance and evade the LUX constraints, but this requirement may be in conflict with the realization of a SFOEWPT except for a finely tuned region in the vicinity of the Higgs pole.

\section{DARK MATTER}

In the cxSM, there are two possibilities for dark matter: (a) a single-component scenario, wherein the pseudoscalar particle $A$ is the dark matter particle and (b) a two-component scenario involving both $A$ and $S$. Scenario (b) is only possible if $v_{S 0}=0$ such as the S2 case. We study the basic dark matter properties in both the $\mathrm{S} 1$ and $\mathrm{S} 2$ cases. $^{7}$ We use micrOMEGAs $[55,56]$ to calculate the relic density of $A, \Omega_{A}$, and its spinindependent scattering cross section with the nucleon $N$, $\sigma_{\mathrm{SI}}^{N}$. To be consistent with observation, the parameters of the cxSM must not yield a relic density larger than the experimental value [57],

$$
\Omega_{\mathrm{DM}} h^{2}=0.1186 \pm 0.0020
$$

\footnotetext{
${ }^{7}$ Note that $H_{2}=S$ in $\mathrm{S} 2$.
} 

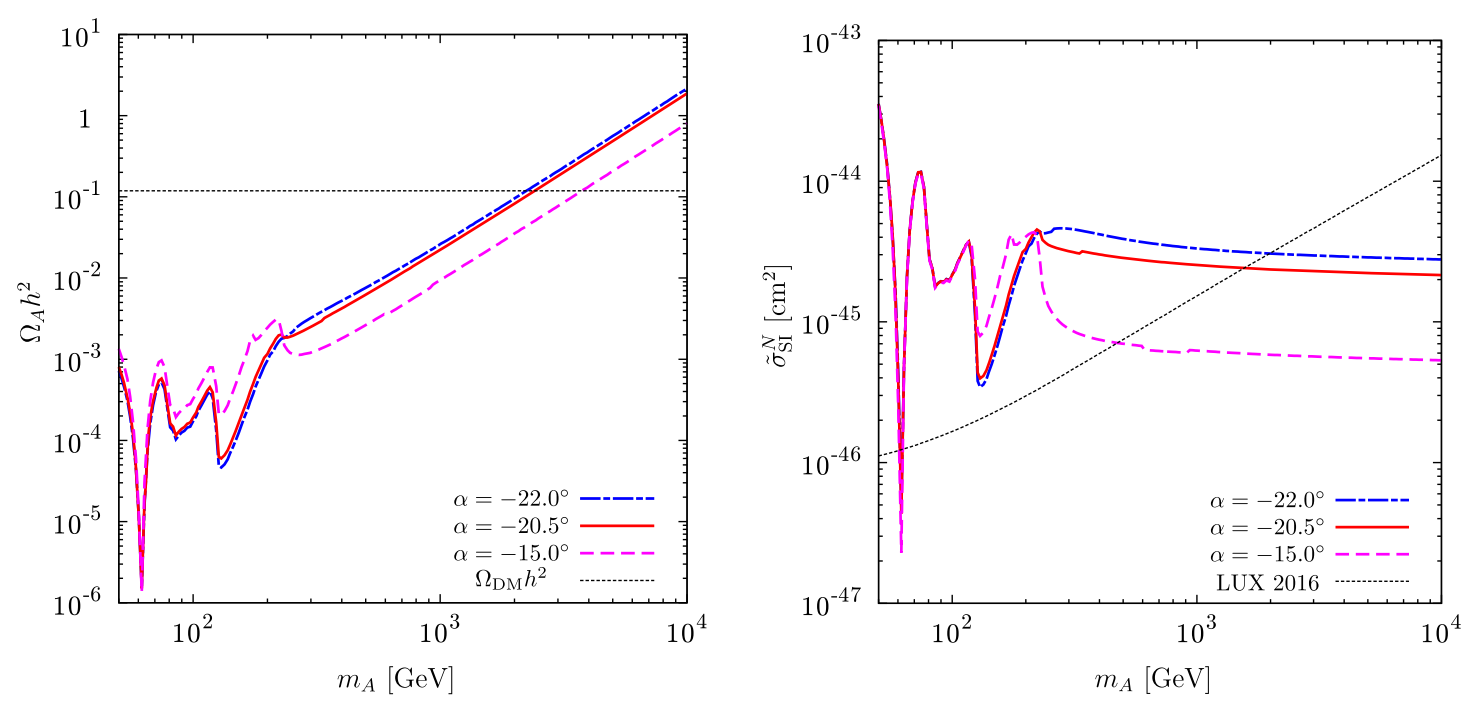

FIG. 11. Scalar dark matter for case S1: (Left) Relic density of $A$ with $\alpha=-22.0^{\circ},-20.5^{\circ}$ and $-15.0^{\circ}$. (Right) Scaled spinindependent DM-nucleon scattering cross section. The allowed region is only at $m_{A} \simeq m_{H_{1}} / 2 \simeq 62.5 \mathrm{GeV}$ if a SFOEWPT is required.

nor should the rate for spin-independent scattering from the nucleon exceed the current LUX limit [36]. For a DM mass of around $50 \mathrm{GeV}$, the maximum spin-independent cross section is $\sigma_{\mathrm{SI}}^{N} \simeq 1.1 \times 10^{-46} \mathrm{~cm}^{2}$, assuming the DM candidate saturates the relic density. ${ }^{8}$

In the present case, if the relic abundance of $A$ (understood to imply the combined $A$ and $S$ abundance in S2) is less than the observed DM abundance, $\sigma_{\mathrm{SI}}^{N}$ should be scaled as

$$
\tilde{\sigma}_{\mathrm{SI}}^{N}=\sigma_{\mathrm{SI}}^{N}\left(\frac{\Omega_{A}}{\Omega_{\mathrm{DM}}}\right)
$$

For the maximum allowed $\Omega_{A} h^{2}$ we use the central value of Eq. (35).

In the left plot of Fig. 11, $\Omega_{A} h^{2}$ is plotted as a function of $m_{A}$ for case S1. We take $\alpha=-22.0^{\circ}$ (blue, dot-dashed), $20.5^{\circ}$ (red, solid) and $-15.0^{\circ}$ (magenta, dashed). $\Omega_{\mathrm{DM}} h^{2}$ is shown by the dotted black line. One can see that $\Omega_{A} h^{2}$ is less than the observed value up to $m_{A} \simeq$ a few $\mathrm{TeV}$, depending on $\alpha$. Note that $\Omega_{A} h^{2}$ is highly suppressed at $m_{A} \simeq m_{H_{1}} / 2$ regardless of $\alpha$, which is due to the resonant enhancement of the annihilation cross section,

$$
\begin{aligned}
\sigma(A A & \left.\rightarrow H_{1,2} \rightarrow X X\right) \\
\propto & \left|\frac{\lambda_{H_{1} A A} g_{H_{1} X X}}{s-m_{H_{1}}^{2}+i m_{H_{1}} \Gamma_{H_{1}}}+\frac{\lambda_{H_{2} A A} g_{H_{2} X X}}{s-m_{H_{2}}^{2}+i m_{H_{2}} \Gamma_{H_{2}}}\right|^{2},
\end{aligned}
$$

\footnotetext{
${ }^{8}$ Recently, the ZENON1T experiment has updated the upper bound on $\sigma_{\mathrm{SI}}^{N}$ [58], which is slightly below the LUX bound. Our conclusions are not substantially affected by the improved limit.
}

where $X$ denotes the gauge bosons or fermions, $\Gamma_{H_{12}}$ are the total widths of $H_{1,2}$, and $s \simeq 4 m_{A}^{2}$. The Higgs couplings are, respectively, given by

$$
\begin{gathered}
\lambda_{H_{1} A A}=\left(\delta_{2} v_{0} \cos \alpha+d_{2} v_{S 0} \sin \alpha\right) / 2, \\
\lambda_{H_{2} A A}=\left(-\delta_{2} v_{0} \sin \alpha+d_{2} v_{S 0} \cos \alpha\right) / 2, \\
g_{H_{1} V V}=g_{H_{1} \bar{f} f}=\cos \alpha, \\
g_{H_{2} V V}=g_{H_{2} \bar{f} f}=-\sin \alpha .
\end{gathered}
$$

Unlike the real singlet DM scenario, one sees more dips in the curves of Fig. 11 due to the contribution of $\mathrm{H}_{2}$.

The right plot shows $\tilde{\sigma}_{\mathrm{SI}}^{N}$ as a function of $m_{A}$. The style and color schemes of the curves are the same as those in the left plot. The dotted black curve is the LUX exclusion bound. The allowed regions are $m_{A} \gtrsim 475 \mathrm{GeV}$ for $\alpha=-15.0^{\circ}$, $m_{A} \gtrsim 1560 \mathrm{GeV}$ for $\alpha=-20.5^{\circ}, \quad m_{A} \gtrsim 1995 \mathrm{GeV}$ for $\alpha=-22.0^{\circ}$, and at $m_{A} \simeq m_{H_{1}} / 2$ for the three cases.

It should be emphasized that even though the large $m_{A}$ regions are consistent with the DM data, the SFOEWPT is not realized since the $T=0$ vacuum energy of phase A is lower than that of phase B in this regime, as mentioned in the last paragraph of the previous section. For $\alpha=-20.5^{\circ}$, the occurrence of a SFOEWPT leads to an upper bound of $m_{A} \lesssim 700 \mathrm{GeV}$.

The quantities $\Omega_{A} h^{2}$ and $\tilde{\sigma}_{\mathrm{SI}}^{N}$ in the case of S2 are shown in the left and right plots of Fig. 12, respectively. In this case, the scalar particle $H_{2}(=S)$ is also stable. We consider the illustrative situation in which $m_{H_{2}}=m_{A}$, and thus 

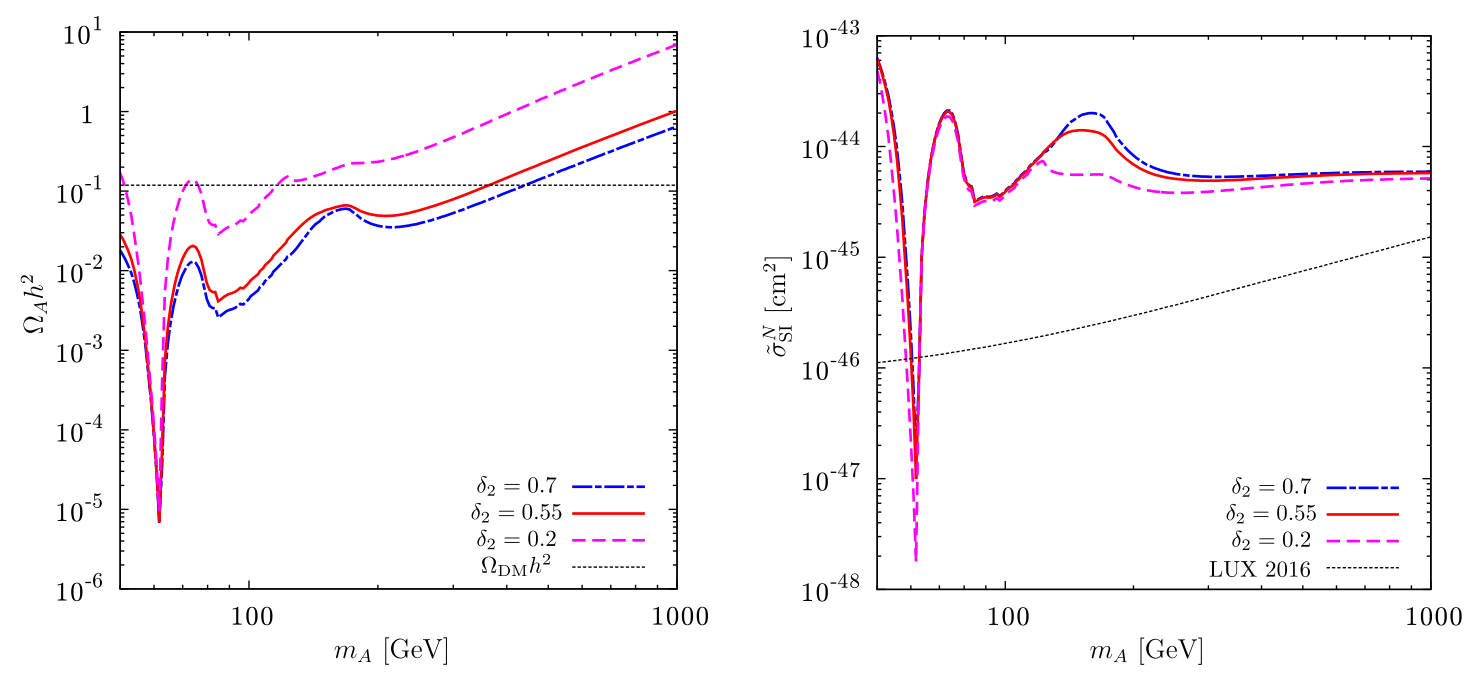

FIG. 12. Scalar dark matter for case S2: (Left) Relic density of $A$ and $S$ with $\delta_{2}=0.7,0.55$ and 0.2 , where $m_{A}=m_{S}$ is assumed. (Right) Scaled spin-independent DM-nucleon scattering cross section.

both particles contribute to the relic density (for a more general discussion of two-component DM in the cxSM, see Refs. [31,59]). We take $\delta_{2}=0.7$ (blue, dot-dashed), 0.55 (red, solid) and 0.2 (magenta, dashed). One can see that a larger $\delta_{2}$ gives a smaller $\Omega_{A} h^{2}$. In contrast to the S1 case, there are regions below $1 \mathrm{TeV}$ for which $\Omega_{A} h^{2}=0.1186$ in all three cases. However, such points are disfavored by the LUX data, as shown in the right plot. The reason why $\mathrm{S} 2$ is more constrained than $\mathrm{S} 1$ by the DM direct detection is that the scaling factor $\Omega_{A} / \Omega_{\mathrm{DM}}$ defined in Eq. (36) is larger than that in $\mathrm{S} 1$. We conclude that only the $m_{A} \simeq 62.5 \mathrm{GeV}$ case is allowed by the LUX data, yet $\Omega_{A} h^{2}$ is well below the observed value.

Now, we comment on the dependence of $m_{A}$ on $T_{C}$, starting with case $\mathrm{S} 2$. To be consistent with the nonzero $\bar{v}_{S}^{\mathrm{A}}\left(T_{C}\right)$ given in Eq. (34), $m_{A}$ must satisfy

$$
m_{A}<\sqrt{\frac{\delta_{2}}{4} v_{0}^{2}-2 \Sigma_{S} T_{C}^{2}}
$$

where the mass formula (10) is used. Our numerical study shows that $m_{A} \lesssim 81.9 \mathrm{GeV}$. In the $\mathrm{S} 1$ case, Eq. (34) does not apply, while its analog is not particularly enlightening. Consequently, we draw entirely upon a numerical exploration of the $m_{A}$-dependence and obtain an upper bound of around $700 \mathrm{GeV}$ for $\alpha=-20.5$.

\section{PHENOMENOLOGICAL DISCUSSIONS AND COMPARISONS WITH A PREVIOUS STUDY}

Let us briefly discuss collider phenomenology of our benchmark scenarios. We do so simply to illustrate the degree to which the parameter space leading to both a viable DM candidate and a SFOEWPT may be probed at the LHC. We defer a comprehensive analysis to a future study. For case $\mathrm{S} 1, H_{2}$ can decay into an $A A$ pair, with the partial width given by

$$
\Gamma_{H_{2} \rightarrow A A}=\frac{\lambda_{H_{2} A A}^{2}}{32 \pi m_{H_{2}}} \sqrt{1-\frac{4 m_{A}^{2}}{m_{H_{2}}^{2}}} .
$$

For $\alpha=-20.5^{\circ}$, we obtain $\operatorname{Br}\left(H_{2} \rightarrow A A\right) \simeq 1$. In the narrow width approximation, this leads to $\sigma\left(g g \rightarrow H_{2}\right)$ $\operatorname{Br}\left(H_{2} \rightarrow A A\right)=s_{\alpha}^{2} \sigma\left(g g \rightarrow H_{1}(230)\right) \operatorname{Br}\left(H_{2} \rightarrow A A\right) \simeq$ 2.36(5.42) pb, where $H_{1}(230)$ denotes a SM-like Higgs boson with the mass $230 \mathrm{GeV}$, and where we have used $\sigma\left(g g \rightarrow H_{1}(230)\right)=5.57(15.1) \mathrm{pb}$ at 8 (13) TeV LHC [60]. With these rates, one could, in principle, search for an invisibly decaying heavy Higgs boson at the LHC. ${ }^{9}$

For case S2, on the other hand, it is difficult to probe at the colliders as it does not have a distinctive signature. This is because $m_{H_{2}}=m_{A}=m_{H_{1}} / 2=62.5 \mathrm{GeV}$ and the signal strengths of $H_{1}$ are the same as in the SM. However, if the DM mass lies slightly below $m_{H_{1}} / 2, H_{1}$ could have an invisible decay mode, which can, in principle, be probed via the vector boson fusion processes. Detailed studies can be found in Refs. [17,31,62,63].

Before concluding, we make a comparison of this work with Ref. [33]. The latter study includes the operators $H^{\dagger} H \mathbb{S}, \mathbb{S}|\mathbb{S}|^{2}$ and their Hermitian conjugates that are not considered in the current work. On the other hand, the tadpole term $\mathbb{S}$ does not exist in Ref. [33]. With those differences in mind, some distinctive conclusions between

\footnotetext{
${ }^{9}$ Since $A$ is the DM, its typical collider signature is a missing transverse momentum recoil against a visible system. For recent Higgs invisible decay searches at the LHC, see, e.g., Ref. [61].
} 
the two studies emerge: in our analysis, (1) the one-step EWPT (O $\rightarrow$ B transition) is absent and (2) there is no parameter space that can accommodate both SFOEWPT and the observed DM relic density simultaneously in the large $m_{A}$ region.

The former may be attributed to the model setup since the operators $H^{\dagger} H \mathbb{S}$ and $\mathbb{S}|\mathbb{S}|^{2}$ play an important role in realizing the one-step EWPT, as argued in Sec. III (see also Ref. [33]). On the other hand, the latter may be due to the method used in analyzing the EWPT. At tree level, the approaches are equivalent. Indeed, as discussed in Ref. [33], $m_{A}$ is irrelevant to the vacuum energy difference between phases A and B at the tree level, which implies that the two-step EWPT is independent of $m_{A}$. Beyond this order, the vacuum energies are no longer independent of $m_{A}$. Consequently, differences in treating the EWPT beyond tree level have implications for the viability of a SFOEWPT as a function of $m_{A}$. Specifically, in our gauge-invariant next-to-leading order calculation, a large $m_{A}$ is not compatible with a SFOEWPT since the $T=0$ vacuum energy of phase A lies below that of phase $\mathrm{B}$ as discussed above. This situation represents a clear difference from the findings in Ref. [33].

\section{CONCLUSIONS}

The complex scalar extension of the SM, or cxSM, is a minimal scenario with the potential to address two issues in cosmology: the generation of a SFOEWPT as needed for EWBG and particle dark matter. With two additional scalar degrees of freedom, it provides an in-principle viable DM candidate as the Goldstone boson of a spontaneously broken global U(1) symmetry (given a mass with soft symmetry-breaking operators), while allowing the remaining scalar to catalyze a SFOEWPT as in the real scalar singlet extensions of the SM. The EWPT and DM dynamics are intertwined, governed by a common set of gaugeinvariant operators.

The cxSM also provides a tractable framework for addressing several theoretical issues that pertain to the dynamics of electroweak symmetry-breaking transition. The purpose of the present study is to determine the degree to which a careful treatment of these issues, enumerated in Sec. I, would affect the viability of the cxSM for both DM and baryogenesis. In general, we find that the use of a RG-improved potential can be implemented in a way that essentially eliminates otherwise problematic scale dependence while allowing for a gauge-invariant treatment of the EWPT. For the cxSM, this gauge-invariant, RG-improved treatment generally leads to a restricted region of the model parameter space that is consistent with the results of DM direct detection experiments and a two-step scenario for a SFOEWPT. For the basis of operators included in our study, the two-step SFOEWPT is viable only for a finely tuned region of parameter space where the DM mass is roughly half that of the SM-like Higgs scalar. It is possible that inclusion of cubic U(1)-breaking operators could enable a single-step SFOEWPT with a wider range of DM masses, though an analysis of this possibility should be revisited using a gauge-invariant, RG-improved framework.

Our study also illustrates the need to exercise caution when applying perturbation theory to EWPT dynamics, a principle that goes beyond the cxSM. ${ }^{10}$ We have observed quantitatively noticeable differences between two gaugeinvariant approaches: the high-temperature effective theory in which one retains only the finite- $T$ quadratic terms, and the $\hbar$-expansion. Use of the latter appears to imply more effective preservation of the baryon asymmetry than does the former. These differences can become more pronounced near the critical temperature as well as near the end points of a first-order transition in various regions of parameter space, regions where one might expect higher order contributions to be relatively more important. The assumption of a $T$-independent proportionality between the leading-order sphaleron energy and the gauge-invariant scale associated with EWSB can also break down in the vicinity of $T_{C}$. We anticipate that these lessons will apply to EWPT dynamics in other models, pointing to the importance of developing more refined perturbative treatments, comparing with nonperturbative computations for representative benchmark parameter choices, and avoiding overly strong conclusions regarding the viability of a given model.

\section{ACKNOWLEDGMENTS}

C. C. and E. S. were supported in part by the Ministry of Science and Technology of Taiwan under Grants No. 104-2628-M-008-004-MY4 and No. 105-2811-M002-126, respectively, and IBS under Grant No. IBSR018-D1 (E. S.). M. J. R.-M. was supported in part by U.S. Department of Energy Contract No. DE-SC0011095. M.J.R.-M. is also grateful for the hospitality of the Department of Physics at the University of Arizona, where a portion of this work was completed.

\section{APPENDIX: SPHALERON SOLUTIONS}

To compute $E_{\text {sph }}(T)$, we obtain the sphaleron solutions following a method suggested in Refs. [65,66] and its extension including a singlet scalar field [44]. We neglect the effects of $\mathrm{U}(1)_{Y}$ as they are less than a few percent $[67,68]$.

\footnotetext{
${ }^{10}$ This point has been emphasized in Ref. [35] and more recently in Ref. [64], which develops a refined thermal resummation method.
} 
A noncontractible loop configuration is given by

$$
U(\mu, \theta, \phi)=\left(\begin{array}{cc}
e^{i \mu}(\cos \mu-i \sin \mu \cos \theta) & e^{i \phi} \sin \mu \sin \theta \\
-e^{-i \phi} \sin \mu \sin \theta & e^{-i \mu}(\cos \mu+i \sin \mu \cos \theta)
\end{array}\right)
$$

where $\mu$ runs from 0 to $\pi$, parametrizing a least energy path between two adjacent topologically distinct vacua. The configuration at $\mu=\pi / 2$ corresponds to the sphaleron.

The gauge and Higgs fields are expressed in terms of the above noncontractible loop configuration as

$$
\begin{gathered}
A_{i}(\mu, r, \theta, \phi)=\frac{i}{g_{2}} f(r) \partial_{i} U(\mu, \theta, \phi) U^{-1}(\mu, \theta, \phi), \\
\Phi(\mu, r, \theta, \phi)=\frac{\bar{v}(T)}{\sqrt{2}}\left[(1-h(r))\left(\begin{array}{c}
0 \\
e^{-i \mu} \cos \mu
\end{array}\right)+h(r) U(\mu, \theta, \phi)\left(\begin{array}{l}
0 \\
1
\end{array}\right)\right], \\
S(\mu, r, \theta, \phi)=\frac{\bar{v}_{S}^{\mathrm{B}}(T)}{\sqrt{2}} k(r),
\end{gathered}
$$

where $\bar{v}$ and $\bar{v}_{S}$ are determined using Eq. (11).

The energy functional in the $A_{0}=0$ gauge takes the form

$$
E_{\mathrm{sph}}[f, h, k ; T]=\frac{4 \pi \Omega(T)}{g_{2}} \mathcal{E}(T),
$$

where

$$
\mathcal{E}(T)=\int_{0}^{\infty} d \xi\left[4 f^{\prime 2}+\frac{8}{\xi^{2}}\left(f-f^{2}\right)^{2}+\frac{\xi^{2}}{2} \frac{v^{2}}{\Omega^{2}} h^{\prime 2}+\frac{v^{2}}{\Omega^{2}} h^{2}(1-f)^{2}+\frac{\xi^{2}}{2} \frac{v_{S}^{2}}{\Omega^{2}} k^{\prime 2}+\frac{\xi^{2}}{g_{2}^{2} \Omega^{4}} V^{\mathrm{HT}}(h, k ; T)\right],
$$

with $\xi=g_{2} \Omega r$. The prime on them denotes a derivative with respect to $\xi$. From the energy functional, one can find the equations of motion for the sphaleron configurations,

$$
\begin{gathered}
\frac{d^{2} f}{d \xi^{2}}=\frac{2}{\xi^{2}}\left(f-f^{2}\right)(1-2 f)-\frac{v^{2} h^{2}}{4 \Omega^{2}}(1-f), \quad \frac{d}{d \xi}\left[\xi^{2} \frac{d h}{d \xi}\right]=2 h(1-f)^{2}+\frac{\xi^{2}}{g_{2}^{2}} \frac{1}{\bar{v}^{2} \Omega^{2}} \frac{\partial V^{\mathrm{HT}}}{\partial h}, \\
\frac{d}{d \xi}\left[\xi^{2} \frac{d k}{d \xi}\right]=\frac{\xi^{2}}{g_{2}^{2}} \frac{1}{\bar{v}_{S}^{2} \Omega^{2}} \frac{\partial V^{\mathrm{HT}}}{\partial k}
\end{gathered}
$$

with the boundary conditions,

$$
\lim _{\xi \rightarrow 0} f(\xi)=0, \quad \lim _{\xi \rightarrow 0} h(\xi)=0, \quad \lim _{\xi \rightarrow 0} k^{\prime}(\xi)=0, \quad \lim _{\xi \rightarrow \infty} f(\xi)=1, \quad \lim _{\xi \rightarrow \infty} h(\xi)=1, \quad \lim _{\xi \rightarrow \infty} k(\xi)=1 .
$$

[1] P. A. R. Ade et al. (Planck Collaboration), Astron. Astrophys. 571, A16 (2014).

[2] A. D. Sakharov, Pisma Zh. Eksp. Teor. Fiz. 5, 32 (1967); A. D. Sakharov, Usp. Fiz. Nauk 161, 61 (1991).

[3] V. Kuzmin, V. Rubakov, and M. Shaposhnikov, Phys. Lett. 155B, 36 (1985).
[4] A. G. Cohen, D. B. Kaplan, and A. E. Nelson, Annu. Rev. Nucl. Part. Sci. 43, 27 (1993).

[5] M. Quiros, Helv. Phys. Acta 67, 451 (1994).

[6] V. A. Rubakov and M. E. Shaposhnikov, Usp. Fiz. Nauk 166, 493 (1996); V. A. Rubakov and M. E. Shaposhnikov, Phys. Usp. 39, 461 (1996). 
[7] K. Funakubo, Prog. Theor. Phys. 96, 475 (1996).

[8] M. Trodden, Rev. Mod. Phys. 71, 1463 (1999).

[9] W. Bernreuther, Lect. Notes Phys. 591, 237 (2002).

[10] J. M. Cline and Baryogenesis, in Les Houches Summer School-Session 86: Particle Physics and Cosmology: The Fabric of Spacetime, Les Houches, France, 2006.

[11] D. E. Morrissey and M. J. Ramsey-Musolf, New J. Phys. 14, 125003 (2012).

[12] T. Konstandin, Phys. Usp. 56, 747 (2013); T. Konstandin, Usp. Fiz. Nauk 183, 785 (2013).

[13] K. Kajantie, M. Laine, K. Rummukainen, and M. E. Shaposhnikov, Phys. Rev. Lett. 77, 2887 (1996).

[14] K. Rummukainen, M. Tsypin, K. Kajantie, M. Laine, and M. E. Shaposhnikov, Nucl. Phys. B532, 283 (1998).

[15] F. Csikor, Z. Fodor, and J. Heitger, Phys. Rev. Lett. 82, 21 (1999).

[16] Y. Aoki, F. Csikor, Z. Fodor, and A. Ukawa, Phys. Rev. D 60, 013001 (1999).

[17] V. Barger, P. Langacker, M. McCaskey, M. J. RamseyMusolf, and G. Shaughnessy, Phys. Rev. D 77, 035005 (2008).

[18] S. Profumo, M. J. Ramsey-Musolf, and G. Shaughnessy, J. High Energy Phys. 08 (2007) 010.

[19] A. Ahriche, Phys. Rev. D 75, 083522 (2007).

[20] A. Noble and M. Perelstein, Phys. Rev. D 78, 063518 (2008).

[21] J. M. Cline, G. Laporte, H. Yamashita, and S. Kraml, J. High Energy Phys. 07 (2009) 040.

[22] J. R. Espinosa, T. Konstandin, and F. Riva, Nucl. Phys. B854, 592 (2012).

[23] J. M. No and M. Ramsey-Musolf, Phys. Rev. D 89, 095031 (2014).

[24] T. Alanne, K. Tuominen, and V. Vaskonen, Nucl. Phys. B889, 692 (2014).

[25] S. Profumo, M. J. Ramsey-Musolf, C. L. Wainwright, and P. Winslow, Phys. Rev. D 91, 035018 (2015).

[26] A. V. Kotwal, M. J. Ramsey-Musolf, J. M. No, and P. Winslow, Phys. Rev. D 94, 035022 (2016).

[27] V. Vaskonen, Phys. Rev. D 95, 123515 (2017).

[28] T. Huang, J. M. No, L. Pernié, M. Ramsey-Musolf, A. Safonov, M. Spannowsky, and P. Winslow, Phys. Rev. D 96, 035007 (2017).

[29] C.-Y. Chen, J. Kozaczuk, and I. M. Lewis, J. High Energy Phys. 08 (2017) 096.

[30] D. Curtin, P. Meade, and C.-T. Yu, J. High Energy Phys. 11 (2014) 127.

[31] V. Barger, P. Langacker, M. McCaskey, M. Ramsey-Musolf, and G. Shaughnessy, Phys. Rev. D 79, 015018 (2009).

[32] M. Gonderinger, H. Lim, and M. J. Ramsey-Musolf, Phys. Rev. D 86, 043511 (2012).

[33] M. Jiang, L. Bian, W. Huang, and J. Shu, Phys. Rev. D 93, 065032 (2016).

[34] F. Sannino and J. Virkajarvi, Phys. Rev. D 92, 045015 (2015).

[35] H. H. Patel and M. J. Ramsey-Musolf, J. High Energy Phys. 07 (2011) 029.

[36] D. S. Akerib et al. (LUX Collaboration), Phys. Rev. Lett. 118, 021303 (2017).
[37] S. A. Abel, S. Sarkar, and P. L. White, Nucl. Phys. B454, 663 (1995).

[38] R. Costa, M. Muhlleitner, M. O. P. Sampaio, and R. Santos, J. High Energy Phys. 06 (2016) 034.

[39] R. Costa, A. P. Morais, M. O. P. Sampaio, and R. Santos, Phys. Rev. D 92, 025024 (2015).

[40] J. R. Espinosa and M. Quiros, Phys. Rev. D 76, 076004 (2007).

[41] S. R. Coleman and E. J. Weinberg, Phys. Rev. D 7, 1888 (1973).

[42] L. Dolan and R. Jackiw, Phys. Rev. D 9, 3320 (1974).

[43] A. D. Linde, Nucl. Phys. B216, 421 (1983); A. D. Linde, Nucl. Phys. B223, 544(E) (1983).

[44] K. Funakubo, A. Kakuto, S. Tao, and F. Toyoda, Prog. Theor. Phys. 114, 1069 (2005).

[45] K. Funakubo and E. Senaha, Phys. Rev. D 79, 115024 (2009).

[46] K. Fuyuto and E. Senaha, Phys. Rev. D 90, 015015 (2014).

[47] A. Ahriche, T. A. Chowdhury, and S. Nasri, J. High Energy Phys. 11 (2014) 096.

[48] K. Fuyuto and E. Senaha, Phys. Lett. B 747, 152 (2015).

[49] N. K. Nielsen, Nucl. Phys. B101, 173 (1975).

[50] R. Fukuda and T. Kugo, Phys. Rev. D 13, 3469 (1976).

[51] V. Khachatryan et al. (CMS Collaboration), J. High Energy Phys. 10 (2015) 144.

[52] J. M. Moreno, M. Quiros, and M. Seco, Nucl. Phys. B526, 489 (1998).

[53] M. E. Carrington and J. I. Kapusta, Phys. Rev. D 47, 5304 (1993).

[54] J. M. Moreno, D. H. Oaknin, and M. Quiros, Nucl. Phys. B483, 267 (1997).

[55] G. Belanger, F. Boudjema, A. Pukhov, and A. Semenov, Comput. Phys. Commun. 176, 367 (2007).

[56] G. Belanger, F. Boudjema, A. Pukhov, and A. Semenov, Comput. Phys. Commun. 185, 960 (2014).

[57] C. Patrignani et al. (Particle Data Group), Chin. Phys. C 40, 100001 (2016).

[58] E. Aprile et al. (XENON Collaboration), Phys. Rev. Lett. 119, 181301 (2017).

[59] S. Bhattacharya, P. Poulose, and P. Ghosh, J. Cosmol. Astropart. Phys. 704 (2017) 043.

[60] J. R. Andersen et al. (LHC Higgs Cross Section Working Group), arXiv:1307.1347.

[61] V. Khachatryan et al. (CMS Collaboration), J. High Energy Phys. 02 (2017) 135.

[62] L. Feng, S. Profumo, and L. Ubaldi, J. High Energy Phys. 03 (2015) 045.

[63] X.-G. He and J. Tandean, J. High Energy Phys. 12 (2016) 074.

[64] D. Curtin, P. Meade, and H. Ramani, arXiv:1612.00466.

[65] N. Manton, Phys. Rev. D 28, 2019 (1983).

[66] F. R. Klinkhamer and N. Manton, Phys. Rev. D 30, 2212 (1984).

[67] F. R. Klinkhamer and R. Laterveer, Z. Phys. C 53, 247 (1992).

[68] M. E. R. James, Z. Phys. C 55, 515 (1992). 